\title{
AN UNCONDITIONALLY STABLE SEMI-IMPLICIT CUTFEM FOR AN INTERACTION PROBLEM BETWEEN AN ELASTIC MEMBRANE AND AN INCOMPRESSIBLE FLUID*
}

\author{
KYLE DUNN ${ }^{\dagger \ddagger}$, ROGER LUI ${ }^{\ddagger}$, AND MARCUS SARKIS ${ }^{\ddagger}$
}

\begin{abstract}
In this paper we introduce a finite element method for the Stokes equations with a massless immersed membrane. This membrane applies normal and tangential forces affecting the velocity and pressure of the fluid. Additionally, the points representing this membrane move with the local fluid velocity. We design and implement a high-accuracy cut finite element method (CutFEM) which enables the use of a structured mesh that is not aligned with the immersed membrane, and we formulate a time discretization that yields an unconditionally energy stable scheme. We prove that the stability is not restricted by the parameter choices that constrained previous finite element immersed boundary methods and illustrate the theoretical results with numerical simulations.
\end{abstract}

Key words. immersed boundary method, finite element method, numerical stability, CutFEM, unfitted methods

AMS subject classifications. $65 \mathrm{~N} 12,65 \mathrm{~N} 30,74 \mathrm{~F} 10$

1. Introduction. Fluid dynamics problems with immersed boundaries have arisen in many real world scenarios such as cardiac blood flow [40, 42] and cell mechanics [2]. Two prevalent ideas are the immersed boundary method introduced by Peskin [41] and the immersed interface method by LeVeque and $\mathrm{Li}$ [31, 32]. These are both finite difference methods developed for very involved problems. We note that the immersed interface method was also extended to the finite element method by imposing flux conservation and continuity of the solution strongly at certain points of $\Gamma$; see $[1,11,17,18,21,22,26,27,33,34]$. In the immersed boundary method, the interface applies a local force when computing the fluid velocity and pressure globally at each time step. The right-hand side function is defined only on the interface and contains a Dirac delta function whose main purpose is to pass information between Eulerian and Lagrangian coordinates. Peskin's use of a finite difference method requires a smoothing of the effects of the force applied by the membrane. Boffi and Gastaldi extended these ideas to the finite element method in [5]. In their work, a variational formulation in weak form is introduced, and the action of the forcing function, due to bending and stretching, is now written as an integral over the immersed membrane. One can also show that when the problem is written in the strong form, the force applied by the membrane to the fluid is equal to the jump in the normal stress [29] of the fluid across the membrane. The conditional energy stability of the method proposed in [5] was proved later in [6].

The framework of our finite element method begins with Nitsche's formulation [39] in order to weakly impose Dirichlet boundary conditions on fitted meshes. In [23], Nitsche's formulation was extended to the case where the domain boundary does not align with the underlying finite element mesh. In our work, we employ one particular fictitious domain finite element method known as CutFEM $[8,9,12,13]$, which allows us to divide the global domain into two non-overlapping subdomains. This technique not only separates the stress on each side, but also allows us to weakly impose a condition on the jump of the normal derivative in lieu of the prescribed forcing function in the earlier work. Our numerical experiments

${ }^{*}$ Received April 18, 2019. Accepted December 8, 2020. Published online on April 13, 2021. Recommended by Roland Herzog. This research was supported by the National Science Foundation Division of Mathematical Sciences Grant No. 1522663 Higher-Order Methods for Interface Problems with Non-Aligned Meshes.

${ }^{\dagger}$ Now at Cold Regions Research and Engineering Laboratory - U.S. Army ERDC, Hanover, NH 03755 (Kyle.G.Dunn@usace.army.mil).

${ }^{\ddagger}$ Mathematical Sciences Department, Worcester Polytechnic Institute, Worcester, MA 01609 ( $\{$ rlui, msarkis\}@wpi.edu). 
show that optimal spatial convergence can be obtained using CutFEM when the interface is described by a static, smooth parameterization.

CutFEM was implemented for the Stokes equation with an immersed boundary and a P1-iso-P2 element in [24], where Hansbo et al. use a known a priori level set method to track the interface. In that article it is noted that the optimality of their approach is independent of the interface representation, which moves with a prescribed velocity. Some additional work has been done on problems with a known interface velocity [25]. Our approach focuses on the movement of the interface not known a priori. In other words, we let the interface move with the velocity of the fluid, which is not known prior to solving the system at each time step. Recently, this approach was considered in [16], wherein the interface was considered as the zero level set of the function that is the solution of a pure advective partial differential equation with the vector field equal to the fluid velocity. Here in this paper, we implement a Q2-P1 time-depedent Stokes element [28, 30, 35, 36, 44] and track the immersed boundary by updating the position of a fixed number of points sampled from the initial curve. One of the contributions of our work here is the semi-implicit discretization of the force term and the corresponding energy estimates for this fluid-membrane problem. As can be seen on the right-hand side of (3.2), the implicitness of a fully-implicit method would appear in two places: a) the position of the interface at time $t_{n+1}$, which is needed for integration, and b) the interface deformation, which depends on the velocity at $t_{n+1}$. The idea of the semi-implicit method is to make the method explicit in a) and implicit via extrapolation in b). This idea can be found in general free-boundary problems such as in [19]; see also [3, 38, 43].

Using techniques similar to those presented in $[3,19,38,43]$, we show that our method is energy stable on the finite difference immersed boundary method. We believe that the method presented in this paper can be extended to time-depedent two-phase flows using results from static interface problems with unfitted meshes for two-phase flows, which has been used by many groups; see, e.g., $[4,9,14,15]$.

This paper is organized as follows. In Section 2 the model is derived, and the strong formulation of the spatially continuous problem is introduced. Then in Section 3 we look into the time discretization of the problem and prove stability results, building to the fully discretized problem. In Section 4 we introduce the necessary notation and spaces of functions en route to defining our finite element methods. We proceed to prove energy stability of the proposed finite element problem, which is unconditional for our semi-implicit method and yields a CFL-like condition for the explicit method. The results of some numerical tests are shown and discussed in Section 5, and we draw conclusions in Section 6.

2. Model formulation. Consider a domain $\Omega$ in $\mathbb{R}^{d}, d=2,3$, which can be any Lipschitz domain. For simplicity, we will define $\Omega:=(0,1)^{2}$. The following equations model an elastic material inside $\Omega$ using the time-dependent incompressible Stokes equations. The stress tensor is defined by $\mathbf{T}:=-\mu \boldsymbol{\varepsilon}(\boldsymbol{u})+\mathbf{I} p$, where $\boldsymbol{\varepsilon}(\boldsymbol{u})=\frac{1}{2}\left(\nabla \boldsymbol{u}+(\nabla \boldsymbol{u})^{T}\right)$ and $p$ is the pressure. To reduce notational clutter, define $\mu$ to be twice the traditional dynamic viscosity. We assume that $\mu>0$ in order to impose proper boundary and interface conditions based on integration by parts of the viscous term. Inside $\Omega$ there will be a closed curve $\Gamma$ representing a massless, elastic interface between two non-overlapping subdomains $\Omega_{1}$ and $\Omega_{2}$. Throughout this paper, we let $\Omega_{1}$ denote the region exterior to the curve $\Gamma$ such that $\partial \Omega_{1}=\partial \Omega \cup \Gamma$ and $\Omega_{2}$ denote the interior region encapsulated by $\Gamma$. Throughout this work, $\mu$ is assumed to be constant. We note that our results, with some minor modifications, hold also for the case where $\mu$ jumps across $\Gamma$ and varies mildly inside each $\Omega_{i}$; see [11, 14, 24] and Remark 4.4.

The description of the interface $\Gamma$ and the model for the jump of the stress across $\Gamma$ are based on the immersed boundary method; see Peskin [41] and Boffi et al. [6]. As we will see in Remark 3.1, it is advantageous to describe $\Gamma$, and therefore the jump of the stress, at time 
$t$ in parametric form $\Gamma(s, t)$ for $s \in[0, L]$ and fixed $L$ independent of time. In general, $s$ is not an arc-length parameterizaton of $\Gamma$ at any time $t$. We use $\mathbf{X}(s, t)$ to denote the Cartesian coordinates of $\Gamma(s, t)$ corresponding to a point $s$ for any given time $t$. Since this is a closed curve, $\mathbf{X}(0, t)=\mathbf{X}(L, t)$ for all time. To construct $\Gamma(s, t)$, we first define $\Gamma(s, 0)$ given by a parametrization $s \in[0, L]$. The fact that $\Gamma$ is not necessarily parameterized by arc-length allows us to define an initial elastic membrane not only with bending, but also with stretching, that is, $|\partial \mathbf{X} / \partial s|$ is not necessarily equal to one. For time $t>0$, we let $\mathbf{X}(s, t)$ be the material point on the elastic membrane that moves from an initial position $\mathbf{X}(s, 0)$, and also we assume that the movement of a point $\mathbf{X}(s, t)$ on the interface is given by the fluid velocity at that point. Hence, we impose continuity of the velocity

$$
\llbracket \boldsymbol{u} \rrbracket=0
$$

on the interface. For a quantity $\phi$ defined over $\Omega$, we denote $\phi_{1}=\left.\phi\right|_{\Omega_{1}}$ and $\phi_{2}=\left.\phi\right|_{\Omega_{2}}$. Then $\llbracket \phi \rrbracket=\left.\left(\phi_{1}-\phi_{2}\right)\right|_{\Gamma}$ denotes the jump of $\phi$ across $\Gamma$ at a given point. We also impose a no-slip condition on the interface, that is,

$$
\frac{\partial \mathbf{X}(s, t)}{\partial t}=\boldsymbol{u}(\mathbf{X}(s, t), t)
$$

The unit tangent vector, chosen to be in the direction of the parameterization, is defined in terms of $s$ by

$$
\boldsymbol{\tau}=\frac{1}{|\partial \mathbf{X} / \partial s|} \frac{\partial \mathbf{X}}{\partial s}
$$

The boundary tension $T(s, t)$ of the elastic membrane is modeled using a generalized Hooke's law, where

$$
T(s, t)=\sigma(|\partial \mathbf{X} / \partial s| ; s, t),
$$

and the function $\sigma$ is defined below. By computing the elastic force on an arbitrary segment between two points $a$ and $b$, we find that

$$
(T \boldsymbol{\tau})(b, t)-(T \boldsymbol{\tau})(a, t)=\int_{a}^{b} \frac{\partial}{\partial s}(T \boldsymbol{\tau})(s, t) d s .
$$

Since this equality holds for any choice of $a$ and $b$, we know the force on $\Gamma$ is defined in terms of $s$ by

$$
\mathbf{F}=\frac{\partial}{\partial s}(T \boldsymbol{\tau})
$$

A slight modification of the proof of [29, Theorem 1] shows that for a force $\mathbf{F}$ defined in terms of $s \in[0, L]$,

$$
\llbracket p \mathbf{n} \rrbracket=-\frac{(\mathbf{F} \cdot \mathbf{n}) \mathbf{n}}{|\partial \mathbf{X} / \partial s|} \quad \mu \llbracket \varepsilon(\boldsymbol{u}) \mathbf{n} \rrbracket=\frac{\mathbf{F}-(\mathbf{F} \cdot \mathbf{n}) \mathbf{n}}{|\partial \mathbf{X} / \partial s|} .
$$

It follows from (2.1) that if we choose $\sigma(|\partial \mathbf{X} / \partial s| ; s, t)$ to be proportional to $|\partial \mathbf{X} / \partial s|$, i.e., $\sigma(|\partial \mathbf{X} / \partial s| ; s, t)=\kappa|\partial \mathbf{X} / \partial s|$, then the jump condition is defined by

$$
\llbracket(\mu \boldsymbol{\varepsilon}(\boldsymbol{u})-p) \mathbf{n} \rrbracket=\frac{\mathbf{F}(s, t)}{\left|\frac{\partial \mathbf{X}}{\partial s}(s, t)\right|}=\frac{\kappa}{\left|\frac{\partial \mathbf{X}}{\partial s}(s, t)\right|} \frac{\partial^{2} \mathbf{X}}{\partial s^{2}}(s, t) .
$$


Physically, (2.2) means that the elastic interface will apply a force as it is stretched or bent at a given point. Here, the jump condition is defined in terms of the respective quantities restricted to $\Gamma(t)$. For example,

$$
\begin{aligned}
\llbracket(\mu \boldsymbol{\varepsilon}(\boldsymbol{u})-p) \mathbf{n} \rrbracket=(( & \left.\mu \boldsymbol{\varepsilon}\left(\boldsymbol{u}_{1}(\mathbf{X}(s, t), t)\right)-p_{1}(\mathbf{X}(s, t), t)\right) \\
& \left.-\left(\mu \varepsilon\left(\boldsymbol{u}_{2}(\mathbf{X}(s, t), t)\right)-p_{2}(\mathbf{X}(s, t), t)\right)\right) \mathbf{n}(\mathbf{X}(s, t), t)
\end{aligned}
$$

for any $s \in[0, L]$. To ease the notation, we denote $\mathbf{n}=\mathbf{n}_{1}$, i.e., the unit normal pointing outward from the exterior $\Omega_{1}$.

We further impose a homogeneous Dirichlet condition on $\partial \Omega$ and homogeneous initial conditions. Combining the Stokes equations with the continuity of velocity and (2.2), the strong form of the equations to be solved is given in Problem 1.

\section{Problem 1: Strong formulation}

Find $\mathbf{X}(s, t), \boldsymbol{u}_{i}(\mathbf{x}, t)$, and $p_{i}(\mathbf{x}, t)$, for $i=1,2$, such that for all $t \in(0, T)$,

$$
\begin{aligned}
\frac{\partial \boldsymbol{u}_{i}}{\partial t}-\mu \nabla \cdot \boldsymbol{\varepsilon}\left(\boldsymbol{u}_{i}\right)+\nabla p_{i} & =0 & & \text { in } \Omega_{i}(t), i=1,2, \\
\nabla \cdot \boldsymbol{u}_{i} & =0 & & \text { in } \Omega_{i}(t), i=1,2, \\
\llbracket(\mu \varepsilon(\boldsymbol{u})-p) \mathbf{n} \rrbracket & =\frac{\kappa}{\left|\frac{\partial \mathbf{X}}{\partial s}\right|} \frac{\partial^{2} \mathbf{X}(s, t)}{\partial s^{2}} & & \text { for } s \in[0, L], \\
\llbracket \boldsymbol{u} \rrbracket & =0 & & \text { for } s \in[0, L], \\
\boldsymbol{u}_{1} & =0 & & \text { on } \partial \Omega, \\
\frac{\partial \mathbf{X}(s, t)}{\partial t} & =\boldsymbol{u}(\mathbf{X}(s, t), t) & & \text { for } s \in[0, L] . \\
\boldsymbol{u} & =0 & & \text { in } \Omega_{i}(0), i=1,2,
\end{aligned}
$$

REMARK 2.1. Note the time dependence of each subdomain and the location of the interface. When deriving the weak formulation, our spaces of test functions depend on time as well.

3. Discrete-time approximation. Given $\Delta t$, we consider equally-spaced time steps $t_{n}=n \Delta t$, for $0 \leq n \leq N_{t}$, where $N_{t}$ is the number of time steps. We also let $\boldsymbol{u}^{n}=$ $\boldsymbol{u}\left(\mathbf{x}, t_{n}\right)$ and $p^{n}=p\left(x, t_{n}\right)$ be the discrete time approximations to the velocity and pressure, respectively, to simplify notation. Then for each $n$, let $\Gamma^{n}=\Gamma\left(t_{n}\right)$ be the interface separating the two subdomains $\Omega_{i}^{n}=\Omega_{i}\left(t_{n}\right)$. By our choice of notation, the location of the interface $\Gamma^{n}$ in discrete time is updated using $\boldsymbol{u}_{i}^{n}$, which has been solved within the subdomain from the previous time step, $\Omega_{i}^{n-1}$. Below in the temporally discrete variations of (2.3a)-(2.3f), we use a backward-difference approximation for $\partial_{t} \boldsymbol{u}_{i}$. In other words, the derivative with respect to time at $t_{n+1}$ is approximated by $\partial_{t} \boldsymbol{u}_{i}^{n+1}=\left(\boldsymbol{u}_{i}^{n+1}-\boldsymbol{u}_{i}^{n}\right) / \Delta t$. We note that $\boldsymbol{u}_{i}^{n}$ is solved in $\Omega_{i}^{n-1}$ and then $\Omega_{i}^{n}$ is obtained by moving $\Gamma\left(t_{n-1}\right)$ with speed $\boldsymbol{u}_{i}^{n}$. Since $\boldsymbol{u}_{i}^{n}$ is obtained in $\Omega_{i}^{n-1}$ and $\boldsymbol{u}_{i}^{n}$ must be integrated over $\Omega_{i}^{n}$ to solve for $\boldsymbol{u}_{i}^{n+1}$, we define

$$
\tilde{\boldsymbol{u}}^{n}:= \begin{cases}\boldsymbol{u}_{1}^{n}(\mathbf{x}), & \text { for } \mathbf{x} \in \Omega_{1}^{n-1} \\ \boldsymbol{u}_{2}^{n}(\mathbf{x}), & \text { for } \mathbf{x} \in \Omega_{2}^{n-1}\end{cases}
$$


Thus, $\tilde{u}^{n}$ will be used in the integration of the backward difference in all temporally discrete weak formulations below; see Problem 2.

REMARK 3.1. In Nitsche's formulation of the interface problem, we must substitute (2.3c) into an integral over $\Gamma(t)$. We note that in its original form, we are integrating with respect to a time-dependent arc length parameterization of the interface. Since (2.3c) is defined in terms of $s$, we transform this integral over $\Gamma(t)$ to an integral over $[0, L]$. We have the following equalities:

$$
\begin{aligned}
(\llbracket(\mu \varepsilon(\boldsymbol{u})-p) \mathbf{n} \rrbracket,\{\boldsymbol{v}\})_{\Gamma(t)} & =\int_{\Omega} \llbracket(\mu \varepsilon(\boldsymbol{u})-p) \mathbf{n} \rrbracket \cdot\{\boldsymbol{v}(\mathbf{x})\} \delta(\mathbf{x}-\mathbf{X}(s, t)) d \mathbf{x} \\
& =\int_{0}^{L} \llbracket(\mu \varepsilon(\boldsymbol{u})-p) \mathbf{n} \rrbracket \cdot\{\boldsymbol{v}(\mathbf{X}(s, t))\}\left|\frac{\partial \mathbf{X}}{\partial s}\right| d s \\
& =-\int_{0}^{L} \kappa \frac{\partial \mathbf{X}}{\partial s}(s, t) \cdot \frac{\partial}{\partial s}\{\boldsymbol{v}(\mathbf{X}(s, t))\} d s
\end{aligned}
$$

where we denote the average of a function $\phi$ by $\{\phi\}=\frac{1}{2}\left(\left.\phi\right|_{\Omega_{1}}+\left.\phi\right|_{\Omega_{2}}\right)$.

The weak formulation can be obtained by the usual integration by parts on (2.3a)-(2.3b) after multiplication by a test function. To symmetrize the problem for increased accuracy and computational efficiency, we add consistent terms to the weak formulation as seen in [10, 23, 24]. A nonsymmetric interior penalty method may also be used; see, e.g., [10, 24].

Recall that each integral over $\Gamma^{n}$ will be expressed in terms of $s$. We also write $\mathbf{X}^{n}(s)=$ $\mathbf{X}\left(s, t_{n}\right)$ to simplify the notation. In the spatially continuous case, we simplify the inner products involving the jump condition on the interface as follows:

- Explicit method:

$$
(\llbracket(\mu \varepsilon(\boldsymbol{u})-p) \mathbf{n} \rrbracket,\{\boldsymbol{v}\})_{\Gamma^{n}}=-\kappa \int_{0}^{L} \frac{\partial \mathbf{X}^{n}(s)}{\partial s} \frac{\partial}{\partial s}\left\{\boldsymbol{v}\left(\mathbf{X}^{n}(s)\right)\right\} d s .
$$

- Semi-implicit method:

$$
\begin{aligned}
& (\llbracket(\mu \varepsilon(\boldsymbol{u})-p) \mathbf{n} \rrbracket,\{\boldsymbol{v}\})_{\Gamma^{n}}=-\kappa \int_{0}^{L} \frac{\partial \mathbf{X}^{n+1}}{\partial s} \frac{\partial}{\partial s}\left\{\boldsymbol{v}\left(\mathbf{X}^{n}(s)\right)\right\} d s \\
& \quad=-\kappa \int_{0}^{L}\left(\frac{\partial \mathbf{X}^{n}}{\partial s}+\Delta t \frac{\partial}{\partial s}\left\{\boldsymbol{u}^{n+1}\left(\mathbf{X}^{n}(s)\right)\right\}\right) \frac{\partial}{\partial s}\left\{\boldsymbol{v}\left(\mathbf{X}^{n}(s)\right)\right\} d s .
\end{aligned}
$$

Observe that the difference between (3.1) and (3.2) is the extrapolation used in the semiimplicit method, where we solve for $\mathbf{X}^{n+1}$ in (2.3f). Note that $\boldsymbol{u}^{n+1}\left(\mathbf{X}^{n}\right)=\left\{\boldsymbol{u}^{n+1}\left(\mathbf{X}^{n}\right)\right\}$ in the spatially continuous problem, and the average is included for comparison to the discrete case. The expression for the forcing function (3.2) incorporates the unknown velocity of the interface at the current time step.

We formulate the continuous-space, discrete-time Problem 2, letting $\mathbf{Y}=\mathbf{X}^{n+1}$ for the semi-implicit method and $\mathbf{Y}=\mathbf{X}^{n}$ for the explicit method. For completeness, we note that the implicit method sets $\mathbf{Y}=\mathbf{X}^{n+1}$ and integrates over $\Omega^{n+1}$ and $\Gamma^{n+1}$ in Problem 2 instead of $\Omega^{n}$ and $\Gamma^{n}$, respectively. Additional challenges arise with the implicit method because $\Gamma^{n+1}$ and $\Omega^{n+1}$ are not known prior to integration, so we omit this discretization. We have included the terms involving $\llbracket \boldsymbol{u}^{n+1} \rrbracket$ in Problem 2 to compare to the one further discretized in space, Problem 3, although $\llbracket \boldsymbol{u}^{n+1} \rrbracket=0$ in the current continuous setting. For the same reason, we include $\left\{\frac{\partial}{\partial s} \boldsymbol{u}^{n+1}\left(\mathbf{X}^{n}(s)\right)\right\}=\frac{\partial}{\partial s} \boldsymbol{u}^{n+1}\left(\mathbf{X}^{n}(s)\right)$. 


\section{Problem 2: Discrete-time weak formulation}

Given $\left(\boldsymbol{u}_{1}^{0}, \boldsymbol{u}_{2}^{0}\right) \in\left[H^{1}\left(\Omega_{1}^{0}\right)\right]^{2} \times\left[H^{1}\left(\Omega_{2}^{0}\right)\right]^{2}$, where

$$
\left.\boldsymbol{u}_{1}^{0}\right|_{\partial \Omega}=0, \quad\left(p_{1}^{0}, p_{2}^{0}\right) \in\left(L^{2}\left(\Omega_{1}^{0}\right) \times L^{2}\left(\Omega_{2}^{0}\right)\right) / \mathbb{R}, \quad \mathbf{X}^{0}:[0, L] \rightarrow \Omega .
$$

Find for all $1 \leq n \leq N_{t}-1$ solutions $\left(\boldsymbol{u}_{1}^{n+1}, \boldsymbol{u}_{2}^{n+1}\right) \in\left[H^{1}\left(\Omega_{1}^{n}\right)\right]^{2} \times\left[H^{1}\left(\Omega_{2}^{n}\right)\right]^{2}$, $\left(p_{1}^{n+1}, p_{2}^{n+1}\right) \in\left(L^{2}\left(\Omega_{1}^{n}\right) \times L^{2}\left(\Omega_{2}^{n}\right)\right) / \mathbb{R}$, and $\mathbf{X}^{n+1}:[0, L] \rightarrow \Omega$ such that

$$
\begin{gathered}
\boldsymbol{u}_{1}^{n+1}=0 \text { on } \partial \Omega, \quad \llbracket \boldsymbol{u}^{n+1} \rrbracket=0 \text { on } \Gamma^{n}, \quad \text { and } \\
\sum_{i=1}^{2} \frac{1}{\Delta t}\left(\boldsymbol{u}_{i}^{n+1}-\tilde{\boldsymbol{u}}^{n}, \boldsymbol{v}_{i}\right)_{\Omega_{i}^{n}}+\mu\left(\boldsymbol{\varepsilon}\left(\boldsymbol{u}_{i}^{n+1}\right), \boldsymbol{\varepsilon}\left(\boldsymbol{v}_{i}\right)\right)_{\Omega_{i}^{n}}-\left(p_{i}^{n+1}, \nabla \cdot \boldsymbol{v}_{i}\right)_{\Omega_{i}^{n}} \\
-\left(\left\{\left(\mu \boldsymbol{\varepsilon}\left(\boldsymbol{u}^{n+1}\right)-p^{n+1}\right) \mathbf{n}\right\}, \llbracket \boldsymbol{v} \rrbracket\right)_{\Gamma^{n}}-\left(\llbracket \boldsymbol{u}^{n+1} \rrbracket,\{\mu \boldsymbol{\varepsilon}(\boldsymbol{v}) \mathbf{n}\}\right)_{\Gamma^{n}} \\
-\left(\left(\mu \boldsymbol{\varepsilon}\left(\boldsymbol{u}_{1}^{n+1}\right)-p_{1}^{n+1}\right) \mathbf{n}, \boldsymbol{v}_{1}\right)_{\partial \Omega}-\left(\boldsymbol{u}_{1}^{n+1}, \mu \boldsymbol{\varepsilon}\left(\boldsymbol{v}_{1}\right) \mathbf{n}\right)_{\partial \Omega} \\
=\int_{0}^{L} \kappa \frac{\partial^{2} \mathbf{Y}}{\partial s^{2}}(s)\left\{\boldsymbol{v}\left(\mathbf{X}^{n}(s)\right)\right\} d s
\end{gathered}
$$

$$
\sum_{i=1}^{2}-\left(\nabla \cdot \boldsymbol{u}_{i}^{n+1}, q_{i}\right)_{\Omega_{i}^{n}}+\left(\llbracket \boldsymbol{u}^{n+1} \rrbracket,\{q \mathbf{n}\}\right)_{\Gamma^{n}}+\left(\boldsymbol{u}_{1}^{n+1}, q_{1} \mathbf{n}\right)_{\partial \Omega}=0
$$

for all $\left(\boldsymbol{v}_{1}, \boldsymbol{v}_{2}\right) \in\left[H^{1}\left(\Omega_{1}^{n}\right)\right]^{2} \times\left[H^{1}\left(\Omega_{2}^{n}\right)\right]^{2}$ and $\left(q_{1}, q_{2}\right) \in L^{2}\left(\Omega_{1}^{n}\right) \times L^{2}\left(\Omega_{2}^{n}\right)$, and

$$
\frac{\mathbf{X}^{n+1}(s)-\mathbf{X}^{n}(s)}{\Delta t}=\left\{\boldsymbol{u}^{n+1}\left(\mathbf{X}^{n}(s)\right)\right\} \quad \text { for } s \in[0, L] .
$$

3.1. Energy estimates. The proposed semi-implicit method combines the analytical simplicity and stability of the implicit method in [6] with the computational convenience of the explicit method. For a quantity $\phi(s)$ defined on $\Gamma$, we define the norm over the reference configuration $R=[0, L]$ to be

$$
\|\phi\|_{L^{2}(R)}^{2}:=\int_{0}^{L}(\phi(s))^{2} d s .
$$

If we define total energy to be the sum of the kinetic and elastic energies

$$
E^{n}:=\frac{1}{2}\left\|\boldsymbol{u}^{n}\right\|_{L^{2}(\Omega)}^{2}+\frac{1}{2} \kappa\left\|\frac{\partial \mathbf{X}^{n}}{\partial s}\right\|_{L^{2}(R)}^{2},
$$

then the following lemma shows that the energy of the system computed using $\mathbf{Y}=\mathbf{X}^{n+1}$ is monotonically decreasing.

LEMMA 3.2. Let $\boldsymbol{u}^{n+1}, p^{n+1}$, and $\mathbf{X}^{n+1}$ be solutions to (3.3a)-(3.3c) at time $t^{n+1}$ with $\mathbf{Y}=\mathbf{X}^{n+1}$. Then the following equality holds:

$$
\begin{aligned}
E^{n+1}=E^{n} & -\frac{1}{2}\left\|\boldsymbol{u}^{n+1}-\boldsymbol{u}^{n}\right\|_{L^{2}(\Omega)}^{2}-\Delta t \sum_{i=1}^{2} \mu\left\|\varepsilon\left(\boldsymbol{u}_{i}^{n+1}\right)\right\|_{L^{2}(\Omega)}^{2} \\
& -\frac{1}{2} \kappa(\Delta t)^{2}\left\|\nabla_{\Gamma} \boldsymbol{u}^{n+1}\right\|_{L^{2}\left(\Gamma^{n}\right)}^{2} .
\end{aligned}
$$


Proof. Begin by letting $\boldsymbol{v}=\boldsymbol{u}^{n+1}$ and $q=p^{n+1}$ in (3.3a)-(3.3b) and subtract (3.3b) from (3.3a), where $\boldsymbol{u}^{n+1}$ stands for $\boldsymbol{u}^{n+1}=\boldsymbol{u}_{i}^{n+1}$ on $\Omega_{i}^{n}$, for $i=1,2$. We note that for each time step we have $\llbracket \boldsymbol{u}^{n+1} \rrbracket=0$ on $\Gamma^{n}$ and $\boldsymbol{u}_{1}^{n+1}=0$ on $\partial \Omega$. Thus, these boundary terms disappear in (3.3a)-(3.3b). Using the symmetry of the bilinear form we are able to simplify the difference of (3.3a) and (3.3b) to

$$
\begin{aligned}
\frac{1}{\Delta t}\left(\boldsymbol{u}^{n+1}-\boldsymbol{u}^{n}, \boldsymbol{u}^{n+1}\right)_{\Omega} & +\mu\left(\varepsilon\left(\boldsymbol{u}^{n+1}\right), \boldsymbol{\varepsilon}\left(\boldsymbol{u}^{n+1}\right)\right)_{\Omega} \\
& +\kappa \int_{0}^{L} \frac{\partial \mathbf{X}^{n+1}}{\partial s}(s) \frac{\partial}{\partial s}\left\{\boldsymbol{u}^{n+1}\left(\mathbf{X}^{n}(s)\right)\right\} d s=0 .
\end{aligned}
$$

First, we can rewrite

$$
\sum_{i=1}^{2} \mu\left(\varepsilon\left(\boldsymbol{u}_{i}^{n+1}\right), \varepsilon\left(\boldsymbol{u}_{i}^{n+1}\right)\right)_{\Omega_{i}^{n}}=\sum_{i=1}^{2} \mu\left\|\varepsilon\left(\boldsymbol{u}_{i}^{n+1}\right)\right\|_{L^{2}\left(\Omega_{i}^{n}\right)}^{2}=\mu\left\|\varepsilon\left(\boldsymbol{u}^{n+1}\right)\right\|_{L^{2}(\Omega)}^{2} .
$$

Now simplifying the forcing term in (3.7), we have

$$
\begin{aligned}
\kappa & \int_{0}^{L} \frac{\partial \mathbf{X}^{n+1}}{\partial s}(s) \frac{\partial}{\partial s}\left\{\boldsymbol{u}^{n+1}\left(\mathbf{X}^{n}(s)\right)\right\} d s \\
& =\frac{\kappa}{\Delta t} \int_{0}^{L} \frac{\partial \mathbf{X}^{n+1}}{\partial s}\left(\frac{\partial \mathbf{X}^{n+1}}{\partial s}-\frac{\partial \mathbf{X}^{n}}{\partial s}\right) d s \\
& =\frac{\kappa}{2 \Delta t} \int_{0}^{L}\left(\frac{\partial \mathbf{X}^{n+1}}{\partial s}+\frac{\partial \mathbf{X}^{n+1}}{\partial s}-\frac{\partial \mathbf{X}^{n}}{\partial s}+\frac{\partial \mathbf{X}^{n}}{\partial s}\right)\left(\frac{\partial \mathbf{X}^{n+1}}{\partial s}-\frac{\partial \mathbf{X}^{n}}{\partial s}\right) d s \\
& =\frac{\kappa}{2 \Delta t} \int_{0}^{L}\left[\left(\frac{\partial \mathbf{X}^{n+1}}{\partial s}\right)^{2}+\left(\frac{\partial \mathbf{X}^{n+1}}{\partial s}-\frac{\partial \mathbf{X}^{n}}{\partial s}\right)^{2}-\left(\frac{\partial \mathbf{X}^{n}}{\partial s}\right)^{2}\right] d s \\
& =\frac{\kappa}{2 \Delta t}\left(\left\|\frac{\partial \mathbf{X}^{n+1}}{\partial s}\right\|_{L^{2}(R)}^{2}+\left\|\frac{\partial \mathbf{X}^{n+1}}{\partial s}-\frac{\partial \mathbf{X}^{n}}{\partial s}\right\|_{L^{2}(R)}^{2}-\left\|\frac{\partial \mathbf{X}^{n}}{\partial s}\right\|_{L^{2}(R)}^{2}\right) \\
& =\frac{\kappa}{2 \Delta t}\left(\left\|\frac{\partial \mathbf{X}^{n+1}}{\partial s}\right\|_{L^{2}(R)}^{2}+\left\|\Delta t \frac{\partial}{\partial s} \boldsymbol{u}^{n+1}\left(\mathbf{X}^{n}\right)\right\|_{L^{2}(R)}^{2}-\left\|\frac{\partial \mathbf{X}^{n}}{\partial s}\right\|_{L^{2}(R)}^{2}\right) \\
& =\frac{\kappa}{2 \Delta t}\left(\left\|\frac{\partial \mathbf{X}^{n+1}}{\partial s}\right\|_{L^{2}(R)}^{2}+\Delta t^{2}\left\|\nabla_{\Gamma} \boldsymbol{u}^{n+1}\right\|_{L^{2}\left(\Gamma^{n}\right)}^{2}-\left\|\frac{\partial \mathbf{X}^{n}}{\partial s}\right\|_{L^{2}(R)}^{2}\right) .
\end{aligned}
$$

Using a similar manipulation for the first term on the left-hand side of (3.7), we obtain by a simple calculation that

$$
\left(\boldsymbol{u}^{n+1}-\boldsymbol{u}^{n}, \boldsymbol{u}^{n+1}\right)_{\Omega}=\frac{1}{2}\left(\left\|\boldsymbol{u}^{n+1}\right\|_{L^{2}(\Omega)}^{2}+\left\|\boldsymbol{u}^{n+1}-\boldsymbol{u}^{n}\right\|_{L^{2}(\Omega)}^{2}-\left\|\boldsymbol{u}^{n}\right\|_{L^{2}(\Omega)}^{2}\right) .
$$

Applying the above simplifications to each term in (3.7) and multiplying by $\Delta t$ we have (3.6). (

We now turn to the explicit method, whose solution must satisfy equations (3.3a)-(3.3c) with $\mathbf{Y}=\mathbf{X}^{n}$. The velocity $\boldsymbol{u}^{n+1}$ and pressure $p^{n+1}$ are computed by explicitly using the interface location $\Gamma^{n}$ and subdomains $\Omega_{i}^{n}$ determined in the previous time step. The energy estimate for the explicit method is similar to that of the semi-implicit method but lacks the 
stabilizing contribution of the extrapolation used to compute the force of the membrane in (3.2). We have the following lemma.

LEMMA 3.3. Let $\boldsymbol{u}^{n+1}, p^{n+1}$, and $\mathbf{X}^{n+1}$ be solutions to (3.3a)-(3.3c) at time $t^{n+1}$ with $\mathbf{Y}=\mathbf{X}^{n}$. Then the following equality holds:

$$
\begin{aligned}
E^{n+1}=E^{n} & -\frac{1}{2}\left\|\boldsymbol{u}^{n+1}-\boldsymbol{u}^{n}\right\|_{L^{2}(\Omega)}^{2}-\Delta t \sum_{i=1}^{2} \mu\left\|\varepsilon\left(\boldsymbol{u}^{n+1}\right)\right\|_{L^{2}(\Omega)}^{2} \\
& +\frac{\kappa}{2}(\Delta t)^{2}\left\|\nabla_{\Gamma} \boldsymbol{u}^{n+1}\right\|_{L^{2}\left(\Gamma^{n}\right)}^{2} .
\end{aligned}
$$

Proof. We begin with the simplification made in the previous proof:

$$
\begin{aligned}
\frac{1}{\Delta t}\left(\boldsymbol{u}^{n+1}-\boldsymbol{u}^{n}, \boldsymbol{u}^{n+1}\right)_{\Omega} & +\mu\left(\varepsilon\left(\boldsymbol{u}^{n+1}\right), \boldsymbol{\varepsilon}\left(\boldsymbol{u}^{n+1}\right)\right)_{\Omega} \\
& +\kappa \int_{0}^{L} \frac{\partial \mathbf{X}^{n}}{\partial s}(s) \frac{\partial}{\partial s}\left\{\boldsymbol{u}^{n+1}\left(\mathbf{X}^{n}(s)\right)\right\}=0 .
\end{aligned}
$$

The proof for the explicit case is identical to the proof in the semi-implicit case with one important difference in the treatment of the final term in (3.9). We have

$$
\begin{aligned}
\kappa \int_{0}^{L} \frac{\partial \mathbf{X}^{n}}{\partial s}(s) \frac{\partial}{\partial s}\left\{\boldsymbol{u}^{n+1}\left(\mathbf{X}^{n}(s)\right)\right\} \\
=\frac{\kappa}{\Delta t} \int_{0}^{L} \frac{\partial \mathbf{X}^{n}}{\partial s}\left(\frac{\partial \mathbf{X}^{n+1}}{\partial s}-\frac{\partial \mathbf{X}^{n}}{\partial s}\right) d s \\
=\frac{\kappa}{2 \Delta t} \int_{0}^{L}\left(\frac{\partial \mathbf{X}^{n+1}}{\partial s}-\frac{\partial \mathbf{X}^{n+1}}{\partial s}+\frac{\partial \mathbf{X}^{n}}{\partial s}+\frac{\partial \mathbf{X}^{n}}{\partial s}\right)\left(\frac{\partial \mathbf{X}^{n+1}}{\partial s}-\frac{\partial \mathbf{X}^{n}}{\partial s}\right) d s \\
=\frac{\kappa}{2 \Delta t} \int_{0}^{L}\left[\left(\frac{\partial \mathbf{X}^{n+1}}{\partial s}\right)^{2}-\left(\frac{\partial \mathbf{X}^{n+1}}{\partial s}-\frac{\partial \mathbf{X}^{n}}{\partial s}\right)^{2}-\left(\frac{\partial \mathbf{X}^{n}}{\partial s}\right)^{2}\right] d s \\
=\frac{\kappa}{2 \Delta t}\left(\left\|\frac{\partial \mathbf{X}^{n+1}}{\partial s}\right\|_{L^{2}(R)}^{2}-\left\|\frac{\partial \mathbf{X}^{n+1}}{\partial s}-\frac{\partial \mathbf{X}^{n}}{\partial s}\right\|_{L^{2}(R)}^{2}-\left\|\frac{\partial \mathbf{X}^{n}}{\partial s}\right\|_{L^{2}(R)}^{2}\right)
\end{aligned}
$$

The simplification of the last term in (3.9) shown above is almost identical to Lemma 3.2, but the important difference is that the middle term in the final line above is negative. Now the energy may not be decreasing.

To make more sense of the norm involving both $\mathbf{X}^{n+1}$ and $\mathbf{X}^{n}$, we can write it in terms of the surface gradient of the velocity on $\Gamma$ as follows:

$$
\begin{aligned}
\frac{\kappa}{2 \Delta t}\left\|\frac{\partial \mathbf{X}^{n+1}}{\partial s}-\frac{\partial \mathbf{X}^{n}}{\partial s}\right\|_{L^{2}(R)}^{2} & =\frac{\kappa}{2 \Delta t}\left\|\Delta t \frac{\partial}{\partial s} \boldsymbol{u}^{n+1}\left(\mathbf{X}^{n}\right)\right\|_{L^{2}(R)}^{2} \\
& =\frac{\kappa \Delta t}{2}\left\|\nabla_{\Gamma} \boldsymbol{u}^{n+1}\right\|_{L^{2}\left(\Gamma^{n}\right)}^{2} .
\end{aligned}
$$

We substitute the final expression into (3.9) along with the simplification of the time-derivative term in the proof of Lemma 3.2 to get (3.8).

We note that for the discrete case, a trace theorem and an inverse inequality can be used for $\left\|\nabla_{\Gamma} \boldsymbol{u}^{n+1}\right\|_{L^{2}\left(\Gamma^{n}\right)}$ to establish conditional stability; see [6]. From now on, we focus only on the unconditionally stable semi-implicit method since the explicit case can be treated similarly. 
4. Discrete-space finite element approximation. The spatial discretization of the problem requires two steps. First, the interface $\Gamma$ is discretized. Recall that we create a mapping from the interval $[0, L]$ to $\Gamma(s, t)$ with $\mathbf{X}(0, t)=\mathbf{X}(L, t)$ that is not necessarily an arc-length parameterization. We choose equally-spaced points $0=s_{0}<s_{1}<\cdots<s_{m}=L$ by letting $\tilde{h}=L / m$ and $s_{j}=j \tilde{h}$. We note that the set of points $\left\{s_{j}\right\}_{j=0}^{m}$ need not be evenly spaced but is chosen so for computational convenience. Then the initial immersed boundary $\Gamma^{0}$ is approximated by a polygon $\Gamma_{\tilde{h}}^{0}$ with $m$ vertices, where the $j$ th vertex is obtained by evaluating $\mathbf{X}_{j}^{0}=\mathbf{X}\left(s_{j}, 0\right)$. While $\left\{s_{j}\right\}_{j=0}^{m}$ may be equally-spaced for computational convenience, $\operatorname{dist}\left(\mathbf{X}^{n}\left(s_{j}\right), \mathbf{X}^{n}\left(s_{j+1}\right)\right)$ may not be uniform. The interface is then approximated by linear segments between the points $\left\{\mathbf{X}_{j}^{n}\right\}$.

Second, we discretize the bulk fluid. The polygonal approximation $\Gamma_{\tilde{h}}^{0}$ divides $\Omega$ into the two approximate subdomains. As the discrete interface moves, these subdomains will change and are denoted by $\Omega_{i, \tilde{h}}^{n}$ at time $t_{n}$. Let $\mathcal{T}_{h}$ partition $\Omega$ into squares with side length $h$. Then the subset of $\mathcal{T}_{h}$ that overlaps each $\Omega_{i, \tilde{h}}^{n}$ is denoted by

$$
\mathcal{T}_{i, h}^{n}:=\left\{K \in \mathcal{T}_{h}: \operatorname{meas}_{2}\left(K \cap \Omega_{i, \tilde{h}}^{n}\right)>0\right\},
$$

where meas ${ }_{d}$ denotes the Lebesgue measure in $d$ dimensions. These sets of elements are further decomposed into two disjoint sets, $\mathcal{T}_{i, h}^{n, I}$ and $\mathcal{T}_{h}^{n, \Gamma}$. We define the set of elements of $\mathcal{T}_{i, h}^{n}$ strictly interior to $\Omega_{i, \tilde{h}}$ by

$$
\mathcal{T}_{i, h}^{n, I}:=\left\{K \in \mathcal{T}_{i, h}^{n}: K \subset \Omega_{i, \tilde{h}}^{n}\right\}
$$

Similarly, the set of elements of $\mathcal{T}_{i, h}^{n}$ whose interior is intersected by the interface $\Gamma_{\tilde{h}}^{n}$ is defined by

$$
\mathcal{T}_{h}^{n, \Gamma}:=\left\{K \in \mathcal{T}_{h}: \operatorname{meas}_{1}\left(K \cap \Gamma_{\tilde{h}}^{n}\right)>0\right\} .
$$

Thus, for each $i$ and $n$ the relationship $\mathcal{T}_{i, h}^{n}=\mathcal{T}_{i, h}^{n, I} \cup \mathcal{T}_{h}^{n, \Gamma}$ holds. Consider the union of all elements in $\mathcal{T}_{i, h}^{n}$; we define the interior of each union to be the extended subdomain $\Omega_{i, h}^{n, e}$. These subdomains $\Omega_{i, h}^{n, e}$ depend on both $\Gamma_{\tilde{h}}^{n}$ and $h$ and can be formally defined by

$$
\Omega_{i, h}^{n, e}:=\operatorname{Int}\left(\bigcup_{K \in \mathcal{T}_{i, h}^{n}} K\right) .
$$

Many approximate quantities depend on $h$ and $\tilde{h}$, although only one is used as a subscript. For example, $\boldsymbol{u}_{i, h}^{n}, p_{i, h}^{n}, \mathcal{T}_{h}^{n, \Gamma}$, and others depend on both $h$ and $\tilde{h}$. The set of points $\left\{\mathbf{X}_{j}^{n}\right\}_{j=0}^{m}$ depends only on $\tilde{h}$, and the polygon will be refined as $\tilde{h}$ decreases for fixed $L$.

It is worth noting that adjacent points on the discrete interface will not form clusters in the dynamic system and need not be manually redistributed. As a set of adjacent points begin to cluster, other points on the interface must be stretched away from one another due to the incompressibility of the fluid inside the interface. The tangential elastic forces generated along the interface by the stress jump condition (2.2) pull the clustering points away from one another to alleviate localized higher tension; see Example 4 below.

4.1. Finite element problem. For each set of elements $\mathcal{T}_{i, h}^{n}$ we define the finite element spaces

$$
\boldsymbol{V}_{i, h}^{n}:=\left\{\boldsymbol{v} \in\left[C^{0}\left(\Omega_{i, h}^{n, e}\right)\right]^{2}:\left.\boldsymbol{v}\right|_{K} \in\left[\mathcal{Q}^{2}(K)\right]^{2}, \forall K \in \mathcal{T}_{i, h}^{n}\right\}
$$



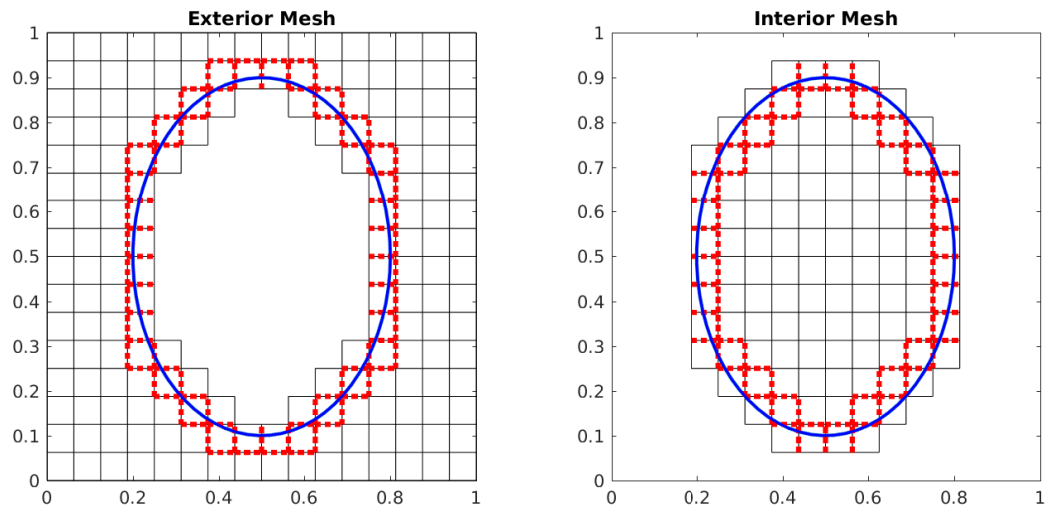

FIG. 4.1. Plot of example meshes with $\mathcal{F}_{i, h}^{n, \Gamma}$ highlighted with a red dotted line for each subdomain.

and

$$
M_{i, h}^{n}:=\left\{q \in L^{2}\left(\Omega_{i, h}^{n, e}\right):\left.q\right|_{K} \in \mathcal{P}^{1}(K), \forall K \in \mathcal{T}_{i, h}^{n}\right\} .
$$

Recall $\mathcal{P}^{1}(K)$ is the space of linear functions defined on an element $K$. A general $q \in M_{i, h}^{n}$ is discontinuous across each edge of the elements since a linear function in two variables is defined by its value at three points. The space $\mathcal{Q}^{2}(K)$ consists of biquadratic functions defined on the element $K$ with nine local degrees of freedom. A general $\boldsymbol{v} \in \boldsymbol{V}_{i, h}^{n}$ has components which are continuous function across the elements.

Additional "ghost" penalty terms are included to mitigate the jumps of the flux and pressure across the faces of elements, particularly to minimize spiking at the ghost nodes and spurious oscillations. To add these to the minimizing functional, we first need to define the sets of edges over which these jumps will be minimized, denoted $\mathcal{F}_{i, h}^{n, \Gamma}$. Informally, we describe each $\mathcal{F}_{i, h}^{n, \Gamma}$ as the union of all edges shared by two elements in $\mathcal{T}_{i, h}^{n}$, where at least one of the elements is in $\mathcal{T}_{h}^{n, \Gamma}$. Formally, these sets are defined by

$$
\mathcal{F}_{i, h}^{n, \Gamma}=\left\{K \cap K^{\prime}: K \neq K^{\prime}, \text { and } K \in \mathcal{T}_{h}^{n, \Gamma}, K^{\prime} \in \mathcal{T}_{i, h}^{n}\right\}
$$

Figure 4.1 shows $\mathcal{F}_{i, h}^{n, \Gamma}$ for each subdomain.

Let $K$ and $K^{\prime}$ be adjacent square elements with $F=K \cap K^{\prime}$ and define $\phi$ on $K$ and $\phi^{\prime}$ on $K^{\prime}$. Below, $[\phi]=\left.\phi\right|_{F}-\left.\phi^{\prime}\right|_{F}$ denotes the jump of a function over the face $F$. Then the stabilizing ghost penalty terms are defined by

$$
\begin{aligned}
j_{i, h}\left(\boldsymbol{u}_{i, h}, \boldsymbol{v}_{i, h}\right) & \left.=\sum_{\ell=0}^{1} \sum_{F \in \mathcal{F}_{i, h}^{n, \Gamma}} \int_{F} h^{2 \ell+1}\left[\partial_{\mathbf{n}_{F}^{(\ell)}}^{(\ell}\left(\boldsymbol{u}_{i, h}\right) \mathbf{n}_{F}\right)\right] \cdot\left[\partial_{\mathbf{n}_{F}}^{(\ell)}\left(\varepsilon\left(\boldsymbol{v}_{i, h}\right) \mathbf{n}_{F}\right)\right], \\
J_{i, h}\left(p_{i, h}, q_{i, h}\right) & =\sum_{\ell=0}^{1} \sum_{F \in \mathcal{F}_{i, h}^{n, \Gamma}} \int_{F} h^{2 \ell+1}\left[\partial_{\mathbf{n}_{F}^{(\ell)}}^{(\ell, h}\right]\left[\partial_{\mathbf{n}_{F}}^{(\ell)} q_{i, h}\right],
\end{aligned}
$$

where $\partial_{\mathbf{n}_{F}}^{(0)} \phi=\phi$ and $\partial_{\mathbf{n}_{F}}^{(1)} \phi$ stands for the derivative of each component of $\phi$ in the $\mathbf{n}_{F}$ direction.

Since the interface cuts through elements, we must weakly impose interface conditions across $\Gamma_{\tilde{h}}^{n}$. The jump of the stress is incorporated naturally by substitution into the integral 
resulting from integration by parts. To impose the weak interface continuity condition and also the weak flux continuity condition, we must add mathematically consistent penalty terms

$$
\gamma_{1} \frac{\mu}{h} \int_{\Gamma_{\bar{h}}^{n}} \llbracket \boldsymbol{u}_{h} \rrbracket \cdot \llbracket \boldsymbol{v}_{h} \rrbracket \quad \text { and } \quad \gamma_{2} \frac{h}{\Delta t} \int_{\Gamma_{\bar{h}}^{n}} \llbracket \boldsymbol{u}_{h} \cdot \mathbf{n} \rrbracket \llbracket \boldsymbol{v}_{h} \cdot \mathbf{n} \rrbracket
$$

for some $\gamma_{1}>0$ and $\gamma_{2}>0$. We note that the second penalty term above is required to establish the inf-sup condition when $h / \Delta t$ dominates $\mu / h$. If $\boldsymbol{u}$ is the exact solution, then the jump of $\boldsymbol{u}$ is equal to zero and (4.1) will vanish for the velocity satisfying the system of equations (2.3a)-(2.3f). Thus, addition of (4.1) will keep the variational formulation consistent with the original problem. We enforce the Dirichlet boundary condition and zero-flux on $\partial \Omega$ by adding the penalty terms

$$
\gamma_{1} \frac{\mu}{h} \int_{\partial \Omega}\left(\boldsymbol{u}_{1, h}-\mathbf{0}\right) \cdot \boldsymbol{v}_{1, h} \quad \text { and } \quad \gamma_{2} \frac{h}{\Delta t} \int_{\partial \Omega}\left(\boldsymbol{u}_{1, h}-\mathbf{0}\right) \cdot \mathbf{n} \boldsymbol{v}_{1, h} \cdot \mathbf{n} .
$$

The parameterization coordinate of the $j$ th vertex of $\Gamma_{\tilde{h}}^{n}$ is denoted $s_{j}$, where $0 \leq j \leq m$, and the corresponding Cartesian coordinate pairs are $\mathbf{X}_{\tilde{h}, j}^{n}=\mathbf{X}_{\tilde{h}}\left(s_{j}, n \Delta t\right)$. Additionally, $s_{0}=0$ and $s_{m}=L$ so that $\mathbf{X}_{\tilde{h}, 0}^{n}=\mathbf{X}_{\tilde{h}, m}^{n}$ for all $n$. To ease the notation, we will let $\mathbf{X}_{j}^{n}$ denote the coordinate pair $\mathbf{X}_{\tilde{h}, j}^{n}$ on the discrete interface.

For both explicit and semi-implicit temporal discretizations, we will find the following simplification of (3.3a) with $\mathbf{Y}=\mathbf{X}^{n}$ useful. After integration by parts on $\Gamma_{\tilde{h}}^{n}$, we simplify the right-hand side of (3.3a) using the fact that $\frac{\partial \mathbf{X}^{n}}{\partial s}$ is constant on each edge of the polygon $\Gamma_{\tilde{h}}^{n}$. If we define

$$
\frac{\partial \mathbf{X}_{j}^{n}}{\partial s}=\frac{\mathbf{X}_{j+1}^{n}-\mathbf{X}_{j}^{n}}{s_{j+1}-s_{j}}
$$

then the resulting simplification is written

$$
\begin{aligned}
-\kappa \int_{0}^{L} \frac{\partial \mathbf{X}^{n}}{\partial s} \frac{\partial}{\partial s}\left\{\boldsymbol{v}\left(\mathbf{X}_{\tilde{h}}^{n}(s)\right)\right\} d s & =-\kappa \sum_{j=0}^{m-1} \frac{\partial \mathbf{X}_{j}^{n}}{\partial s} \int_{s_{j}}^{s_{j+1}} \frac{\partial}{\partial s}\left\{\boldsymbol{v}\left(\mathbf{X}_{\tilde{h}}^{n}(s)\right)\right\} d s \\
& =-\kappa \sum_{j=0}^{m-1} \frac{\partial \mathbf{X}_{j}^{n}}{\partial s}\left(\left\{\boldsymbol{v}\left(\mathbf{X}_{j+1}^{n}\right)\right\}-\left\{\boldsymbol{v}\left(\mathbf{X}_{j}^{n}\right)\right\}\right) \\
& =\kappa \sum_{j=0}^{m-1}\left(\frac{\partial \mathbf{X}_{j+1}^{n}}{\partial s}-\frac{\partial \mathbf{X}_{j}^{n}}{\partial s}\right)\left\{\boldsymbol{v}\left(\mathbf{X}_{j+1}^{n}\right)\right\}
\end{aligned}
$$

To simplify notation in Problem 3 we drop the " $h$ " or " $\tilde{h}$ " subscript from the discrete approximation of the subdomains $\Omega_{h, i}^{n}$, and the quantities $\boldsymbol{u}_{h}^{n}, \boldsymbol{v}_{h}^{n}, p_{h}^{n}, q_{h}^{n}$, and $\mathbf{X}_{\tilde{h}}^{n}$. The definition of $\gamma_{1}, \gamma_{2}, \gamma_{\boldsymbol{u}}$, and $\gamma_{p}$ are given later in the paper; see (4.10).

REMARK 4.1. To distinguish the difference between the explicit and semi-implicit methods in Problem 3, we define a parameter $\nu$ which can be set to either 0 or 1 . Setting $\nu=1$ yields the semi-implicit method, while $\nu=0$ leaves us with the explicit method; see the last term of the left-hand side of (4.4b). We note that for the numerical tests we consider both cases. 


\section{Problem 3: Discrete-time finite element formulation}

1. Solve for $\boldsymbol{u}_{i}^{n+1}$ and $p_{i}^{n+1}$ in

$$
\begin{aligned}
\text { (4.4a) }\left\langle\mathbf{F}^{n+1}, \boldsymbol{v}\right\rangle=\kappa \sum_{j=0}^{m-1}\left(\frac{\partial \mathbf{X}_{j+1}^{n}}{\partial s}-\frac{\partial \mathbf{X}_{j}^{n}}{\partial s}\right)\left\{\boldsymbol{v}^{n+1}\left(\mathbf{X}_{j+1}^{n}\right)\right\} \\
\sum_{i=1}^{2} \frac{1}{\Delta t}\left(\boldsymbol{u}_{i}^{n+1}-\tilde{\boldsymbol{u}}_{i}^{n}, \boldsymbol{v}_{i}\right)_{\Omega_{i}^{n}}+\left(\mu \varepsilon\left(\boldsymbol{u}_{i}^{n+1}\right), \boldsymbol{\varepsilon}\left(\boldsymbol{v}_{i}\right)\right)_{\Omega_{i}^{n}}-\left(p_{i}^{n+1}, \nabla \cdot \boldsymbol{v}_{i}\right)_{\Omega_{i}^{n}} \\
\quad-\left(\left\{\left(\mu \varepsilon\left(\boldsymbol{u}^{n+1}\right)-p^{n+1}\right) \mathbf{n}\right\}, \llbracket \boldsymbol{v} \rrbracket\right)_{\Gamma_{\tilde{h}}^{n}}-\left(\llbracket \boldsymbol{u}^{n+1} \rrbracket,\{\mu \varepsilon(\boldsymbol{v}) \mathbf{n}\}\right)_{\Gamma_{\tilde{h}}^{n}} \\
\quad-\left(\left(\mu \varepsilon\left(\boldsymbol{u}_{1}^{n+1}\right)-p_{1}^{n+1}\right) \mathbf{n}, \boldsymbol{v}_{1}\right)_{\partial \Omega}-\left(\boldsymbol{u}_{1}^{n+1}, \mu \varepsilon\left(\boldsymbol{v}_{1}\right) \mathbf{n}\right)_{\partial \Omega} \\
(4.4 \mathrm{~b}) \quad+\gamma_{\boldsymbol{u}} j_{i, h}^{n}\left(\boldsymbol{u}_{i}^{n+1}, \boldsymbol{v}_{i}\right)+\gamma_{1} \frac{\mu}{h}\left(\llbracket \boldsymbol{u}^{n+1} \rrbracket, \llbracket \boldsymbol{v} \rrbracket\right)_{\Gamma_{\tilde{h}}^{n}}+\gamma_{1} \frac{\mu}{h}\left(\boldsymbol{u}_{1}^{n+1}, \boldsymbol{v}_{1}\right)_{\partial \Omega} \\
\quad+\gamma_{2} \frac{h}{\Delta t}\left(\llbracket \boldsymbol{u}^{n+1} \cdot \mathbf{n} \rrbracket, \llbracket \boldsymbol{v} \cdot \mathbf{n} \rrbracket\right)_{\Gamma_{\tilde{h}}^{n}}+\gamma_{2} \frac{h}{\Delta t}\left(\boldsymbol{u}_{1}^{n+1} \cdot \mathbf{n}, \boldsymbol{v}_{1} \cdot \mathbf{n}\right)_{\partial \Omega} \\
\quad+\nu \kappa \Delta t \int_{\Gamma_{\tilde{h}}} \frac{\partial \boldsymbol{u}^{n+1}\left(\mathbf{X}^{n}\right)}{\partial s} \frac{\partial}{\partial s}\left\{\boldsymbol{v}\left(\mathbf{X}^{n}(s)\right)\right\} d s=\left\langle\mathbf{F}^{n+1}, \boldsymbol{v}\right\rangle \\
\text { (4.4c) } \quad \sum_{i=1}^{2} \quad-\left(\nabla \cdot \boldsymbol{u}_{i}^{n+1}, q_{i}\right)_{\Omega_{i}^{n}}+\left(\llbracket \boldsymbol{u}^{n+1} \rrbracket,\{q \mathbf{n}\}\right)_{\Gamma_{\tilde{h}}^{n}}+\left(\boldsymbol{u}_{1}^{n+1}, q_{1} \mathbf{n}\right)_{\partial \Omega} \\
\quad-\gamma_{p} J_{i, h}^{n}\left(p_{i}^{n+1}, q_{i}\right)=0
\end{aligned}
$$

for all $\boldsymbol{v}_{i}^{n+1} \in \boldsymbol{V}_{i, h}^{n}$ and $q_{i}^{n+1} \in M_{i, h}^{n}, i=1,2$.

2. Solve for $\mathbf{X}_{j}^{n+1}$ using

$$
\mathbf{X}_{j}^{n+1}=\mathbf{X}_{j}^{n}+\Delta t\left\{\boldsymbol{u}^{n+1}\left(\mathbf{X}_{j}^{n}\right)\right\} \quad \text { for } j=0, \ldots, m .
$$

4.2. Energy stability of the FEM. We are able to prove the unconditional stability of the semi-implicit method in Problem 3 under the assumption below, similar to that seen in [37].

Assumption 1. Given $K \in \mathcal{T}_{h}^{n, \Gamma}$, there exists $K^{\prime} \in \mathcal{T}_{i, h}^{n, I}$, an integer $N>0$, and $N$ elements $\left\{K_{k}\right\}_{k=1}^{N}$ such that $K_{1}=K, K_{N}=K^{\prime}$, and $K_{k} \cap K_{k+1} \subset \mathcal{F}_{i, h}^{n, \Gamma}$.

The next lemma is necessary to bound the strain on the extended subdomain $\Omega_{i, h}^{n, e}$ by the strain on the original subdomain $\Omega_{i, \tilde{h}}^{n}$. The result shows why it is necessary to include $j_{i, h}(\boldsymbol{u}, \boldsymbol{v})$ for stability. Here and in the rest of the paper we do not use the fact that the mesh is structured.

Lemma 4.2. Suppose $\boldsymbol{v} \in \boldsymbol{V}_{i, h}^{n}$ is continuous on $\Omega_{i, h}^{n, e}$ and Assumption 1 holds for $\mathcal{T}_{i, h}^{n}$. Then we have the following estimate:

$$
\|\boldsymbol{\varepsilon}(\boldsymbol{v})\|_{L^{2}\left(\Omega_{i, h}^{n, e}\right)}^{2} \leq C_{\boldsymbol{\varepsilon}}\left(\|\boldsymbol{\varepsilon}(\boldsymbol{v})\|_{L^{2}\left(\Omega_{i, \tilde{h}}^{n}\right)}^{2}+j_{i, h}(\boldsymbol{v}, \boldsymbol{v})\right),
$$

where $C_{\varepsilon}$ depends on neither $\boldsymbol{v}$ nor $h$.

Proof. Let $K_{1}$ and $K_{2}$ be neighboring square elements with a shared edge $F=K_{1} \cap K_{2}$. Note that $\|\varepsilon(\boldsymbol{v})\|_{L^{2}\left(\Omega_{i, h}^{n, e}\right)}^{2}$ and $j_{i, h}(\boldsymbol{v}, \boldsymbol{v})$ do not depend on the directions of the Cartesian coordinate axes in which we represent $\boldsymbol{v}$. Therefore, without loss of generality, we assume 
$\mathbf{n}_{F}=(1,0)$ and $\boldsymbol{v}=(u, v)$, and their derivatives are using the canonical representation $x$ and $y$-axis. We make use of the following result from [37, Lemma 5.1]:

$$
\|v\|_{L^{2}\left(K_{1}\right)}^{2} \leq C_{m}\left(\|v\|_{L^{2}\left(K_{2}\right)}^{2}+\sum_{0 \leq \ell \leq p} h^{2 \ell+1} \int_{F}\left|\left[\partial_{\mathbf{n}_{F}}^{\ell} v\right]\right|^{2}\right),
$$

where $\left.v\right|_{K_{1}},\left.v\right|_{K_{2}}$ are polynomial functions of degree less than or equal to $p$. With $\boldsymbol{V}_{i, h}^{n}$ defined as above, the summation in (4.5) simplifies to $p=1$. Denoting $\boldsymbol{v}=(u, v)$, we apply the inequality (4.5) directly to each term of $\boldsymbol{\varepsilon}(\boldsymbol{v}): \boldsymbol{\varepsilon}(\boldsymbol{v})=\left(\partial_{x} u\right)^{2}+\frac{1}{2}\left(\partial_{x} v+\partial_{y} u\right)^{2}+\left(\partial_{y} v\right)^{2}$ and add the inequalities. Note that on any vertical edge, the jump of all $y$-derivatives will be zero because $\boldsymbol{v}$ is continuous across $F$ and $\left.\boldsymbol{v}\right|_{F}$ is simply a polynomial in each component. Also on a vertical edge, the unit normal vector is $\mathbf{n}_{F}=(1,0)$, and it follows that

$$
\left[\partial_{\mathbf{n}_{F}}^{\ell}\left(\varepsilon(\boldsymbol{v}) \mathbf{n}_{F}\right)\right] \cdot\left[\partial_{\mathbf{n}_{F}}^{\ell}\left(\varepsilon(\boldsymbol{v}) \mathbf{n}_{F}\right)\right]=\left(\left[\partial_{x}^{\ell} \partial_{x} u\right]\right)^{2}+\frac{1}{4}\left(\left[\partial_{x}^{\ell}\left(\partial_{x} v+\partial_{y} u\right)\right]\right)^{2} .
$$

The resulting inequality is

$$
\begin{aligned}
\|\varepsilon(\boldsymbol{v})\|_{L^{2}\left(K_{1}\right)}^{2} & =\int_{K_{1}}\left(\partial_{x} u\right)^{2}+\frac{1}{2}\left(\partial_{x} v+\partial_{y} u\right)^{2}+\left(\partial_{y} v\right)^{2} \\
\leq & C_{m}\left(\int_{K_{2}}\left(\partial_{x} u\right)^{2}+\frac{1}{2}\left(\partial_{x} v+\partial_{y} u\right)^{2}+\left(\partial_{y} v\right)^{2}\right. \\
& \left.\quad+\sum_{\ell=0}^{1} h^{2 \ell+1} \int_{F}\left[\partial_{\mathbf{n}_{F}}^{\ell} \partial_{x} u\right]^{2}+\frac{1}{2}\left[\partial_{\mathbf{n}_{F}}^{\ell}\left(\partial_{x} v+\partial_{y} u\right)\right]^{2}\right) \\
\leq & 2 C_{m}\left(\|\varepsilon(\boldsymbol{v})\|_{L^{2}\left(K_{2}\right)}^{2}+\sum_{\ell=0}^{1} h^{2 \ell+1} \int_{F}\left[\partial_{\mathbf{n}_{F}}^{\ell}\left(\varepsilon(\boldsymbol{v}) \mathbf{n}_{F}\right)\right]^{2}\right) .
\end{aligned}
$$

Similarly for any horizontal edge, we let $\mathbf{n}_{F}=(0,1)$ and use the fact that $\left[\partial_{x} u\right]=0$ to get the same inequality.

Using Assumption 1, we are able to find a sequence of at most $N$ adjacent elements leading from an element $K_{1} \in \mathcal{T}_{h}^{n, \Gamma}$ to an element $K_{N} \in \mathcal{T}_{i, h}^{n, I}$. Applying the above inequality across each of the edges $F_{k}=K_{k} \cap K_{k+1}$, for $1 \leq k \leq N-1$, we have

$$
\|\boldsymbol{\varepsilon}(\boldsymbol{v})\|_{L^{2}\left(K_{1}\right)}^{2} \leq\left(2 C_{m}\right)^{N}\left(\|\boldsymbol{\varepsilon}(\boldsymbol{v})\|_{L^{2}\left(K_{N}\right)}^{2}+\sum_{k=1}^{N} \sum_{\ell=0}^{1} \int_{F_{k}}\left[\partial_{\mathbf{n}_{F_{k}}^{\ell}}^{\ell} \boldsymbol{\varepsilon}(\boldsymbol{v}) \mathbf{n}_{F_{k}}\right]^{2}\right) .
$$

Repeating (4.6) for all $K \in \mathcal{T}_{h}^{n, \Gamma}$ and denoting $C_{\varepsilon}=\left(2 C_{m}\right)^{N}$ completes the proof.

Recall the definition of the norm over the reference configuration $R=[0, L]$. For a quantity $\phi(s)$ that is constant on each linear segment of the polygonal $\Gamma_{\tilde{h}}^{n}$, we can simplify (3.4) to

$$
\|\phi\|_{L^{2}(R)}^{2}=\sum_{j=0}^{m-1}\left(\phi\left(s_{j}\right)\right)^{2}\left(s_{j+1}-s_{j}\right)
$$

Above, $\phi\left(s_{j}\right)$ denotes the value of $\phi(s)$ on the interval $\left(s_{j}, s_{j+1}\right)$. Using the definition of the energy (3.5), we are able to prove the following theorem. 
THEOREM 4.3. Let $\boldsymbol{u}_{i, h}^{n+1}, p_{i, h}^{n+1}$, and $\mathbf{X}_{\tilde{h}}^{n+1}$ be solutions to (4.4a)-(4.4d) at time $t^{n+1}$ with $\nu=1$ and assume Assumption 1 holds. Then the following inequality holds:

$$
\begin{aligned}
E^{n+1} \leq E^{n} & -\frac{1}{2}\left\|\boldsymbol{u}_{h}^{n+1}-\boldsymbol{u}_{h}^{n}\right\|_{L^{2}(\Omega)}^{2}+\frac{1}{2} \kappa\left\|\frac{\partial \mathbf{X}_{\tilde{h}}^{n+1}}{\partial s}-\frac{\partial \mathbf{X}_{\tilde{h}}^{n}}{\partial s}\right\|_{L^{2}(R)}^{2} \\
& +\Delta t\left(\gamma_{1} \frac{\mu}{h} \int_{\Gamma_{\tilde{h}}^{n}} \llbracket \boldsymbol{u}_{h}^{n+1} \rrbracket^{2} d s+\gamma_{1} \frac{\mu}{h} \int_{\partial \Omega}\left(\boldsymbol{u}_{1, h}^{n+1}\right)^{2} d s\right. \\
& +\gamma_{2} \frac{h}{\Delta t} \int_{\Gamma_{\tilde{h}}^{n}} \llbracket \boldsymbol{u}_{h}^{n+1} \cdot \mathbf{n} \rrbracket^{2} d s+\gamma_{2} \frac{h}{\Delta t} \int_{\partial \Omega}\left(\boldsymbol{u}_{1, h}^{n+1} \cdot \mathbf{n}\right)^{2} d s \\
& \left.+\sum_{i=1}^{2}\left(\gamma_{\boldsymbol{u}} j_{i, h}\left(\boldsymbol{u}_{i, h}^{n+1}, \boldsymbol{u}_{i, h}^{n+1}\right)+\gamma_{p} J_{i, h}\left(p_{i, h}^{n+1}, p_{i, h}^{n+1}\right)\right)\right) .
\end{aligned}
$$

Proof. We first let $\boldsymbol{v}_{h}=\boldsymbol{u}_{h}^{n+1}$ and $q_{h}=p_{h}^{n+1}$ in (4.4a)-(4.4c) and subtract (4.4c) from (4.4b). After cancellation due to the symmetry of the $L^{2}$-inner product and some simplification we have

$$
\begin{aligned}
\sum_{i=1}^{2} \frac{1}{\Delta t}\left(\boldsymbol{u}_{i, h}^{n+1}-\right. & \left.\boldsymbol{u}_{i, h}^{n}, \boldsymbol{u}_{i, h}^{n+1}\right)_{\Omega_{i, \tilde{h}}^{n}}+\sum_{i=1}^{2} \mu\left\|\varepsilon\left(\boldsymbol{u}_{i, h}^{n+1}\right)\right\|_{L^{2}\left(\Omega_{i, \tilde{h}}^{n}\right)} \\
& -2\left(\llbracket \boldsymbol{u}_{h}^{n+1} \rrbracket,\left\{\mu \varepsilon\left(\boldsymbol{u}_{h}^{n+1}\right) \mathbf{n}\right\}\right)_{\Gamma_{\tilde{h}}^{n}}-2\left(\boldsymbol{u}_{1, h}^{n+1}, \mu \varepsilon\left(\boldsymbol{u}_{1, h}^{n+1}\right) \mathbf{n}_{1}\right)_{\partial \Omega} \\
& +\gamma_{\boldsymbol{u}} j_{h}\left(\boldsymbol{u}_{i, h}^{n+1}, \boldsymbol{u}_{i, h}^{n+1}\right)+\gamma_{p} J_{h}\left(p_{i, h}^{n+1}, p_{i, h}^{n+1}\right) \\
& +\gamma_{1} \frac{\mu}{h} \int_{\Gamma_{\tilde{h}}^{n}} \llbracket \boldsymbol{u}_{h}^{n+1} \rrbracket^{2}+\gamma_{1} \frac{\mu}{h} \int_{\partial \Omega}\left(\boldsymbol{u}_{1, h}^{n+1}\right)^{2} \\
& +\gamma_{2} \frac{h}{\Delta t} \int_{\Gamma_{\tilde{h}}^{n}} \llbracket \boldsymbol{u}_{h}^{n+1} \rrbracket^{2}+\gamma_{2} \frac{h}{\Delta t} \int_{\partial \Omega}\left(\boldsymbol{u}_{1, h}^{n+1}\right)^{2} \\
=\kappa \sum_{j=0}^{m-1} & \left(\frac{\partial \mathbf{X}_{j+1}^{n+1}}{\partial s}-\frac{\partial \mathbf{X}_{j}^{n+1}}{\partial s}\right)\left\{\boldsymbol{u}_{h}^{n+1}\left(\mathbf{X}_{j+1}^{n}\right)\right\}
\end{aligned}
$$

The term on the right-hand side of (4.8) is obtained using (4.3) and (4.4d) as follows:

$$
\begin{aligned}
\kappa \sum_{j=0}^{m-1}\left(\frac{\partial \mathbf{X}_{j+1}^{n+1}}{\partial s}-\frac{\partial \mathbf{X}_{j}^{n+1}}{\partial s}\right)\left\{\boldsymbol{u}_{h}^{n+1}\left(\mathbf{X}_{j+1}^{n}\right)\right\} \\
=-\kappa \int_{0}^{L} \frac{\partial \mathbf{X}^{n+1}}{\partial s} \frac{\partial}{\partial s}\left\{\boldsymbol{u}_{h}^{n+1}\left(\mathbf{X}_{\tilde{h}}^{n}(s)\right)\right\} d s \\
=-\kappa \int_{0}^{L} \frac{\partial \mathbf{X}^{n}}{\partial s} \frac{\partial}{\partial s}\left\{\boldsymbol{u}_{h}^{n+1}\left(\mathbf{X}_{\tilde{h}}^{n}(s)\right)\right\} d s \\
\quad-\kappa \Delta t \int_{0}^{L}\left\{\frac{\partial \boldsymbol{u}_{h}^{n+1}\left(\mathbf{X}^{n}\right)}{\partial s}\right\} \cdot\left\{\frac{\partial \boldsymbol{u}_{h}^{n+1}\left(\mathbf{X}^{n}\right)}{\partial s}\right\} d s \\
\quad-\frac{\kappa \Delta t}{2} \int_{j=0}^{L-1}\left\{\frac{\partial \mathbf{X}_{j+1}^{n}}{\partial s}-\frac{\partial \mathbf{X}_{j}^{n}}{\partial s}\right)\left\{\boldsymbol{u}_{h}^{n+1}\left(\mathbf{X}_{j+1}^{n}\right)\right\} \\
\partial s
\end{aligned}
$$


Following the same simplification as in Lemma 3.2 we have

$$
\sum_{i=1}^{2}\left(\boldsymbol{u}_{i, h}^{n+1}-\boldsymbol{u}_{i, h}^{n}, \boldsymbol{u}_{i, h}^{n+1}\right)_{\Omega_{i, \tilde{h}}^{n}}=\frac{1}{2}\left(\left\|\boldsymbol{u}_{h}^{n+1}\right\|_{L^{2}(\Omega)}^{2}+\left\|\boldsymbol{u}_{h}^{n+1}-\boldsymbol{u}_{h}^{n}\right\|_{L^{2}(\Omega)}^{2}-\left\|\boldsymbol{u}_{h}^{n}\right\|_{L^{2}(\Omega)}^{2}\right)
$$

and

$$
\begin{gathered}
\kappa \sum_{j=0}^{m-1}\left(\frac{\partial \mathbf{X}_{j+1}^{n+1}}{\partial s}-\frac{\partial \mathbf{X}_{j}^{n+1}}{\partial s}\right)\left\{\boldsymbol{u}_{h}^{n+1}\left(\mathbf{X}_{j+1}^{n}\right)\right\} \\
=\frac{\kappa}{2 \Delta t}\left(\left\|\frac{\partial \mathbf{X}_{\tilde{h}}^{n+1}}{\partial s}\right\|_{L^{2}(R)}^{2}+\left\|\frac{\partial \mathbf{X}_{\tilde{h}}^{n+1}}{\partial s}-\frac{\partial \mathbf{X}_{\tilde{h}}^{n}}{\partial s}\right\|_{L^{2}(R)}^{2}-\left\|\frac{\partial \mathbf{X}_{\tilde{h}}^{n}}{\partial s}\right\|_{L^{2}(R)}^{2}\right) .
\end{gathered}
$$

Now we focus on controlling the integrals over the boundaries. First, using the CauchySchwarz inequality and a generalized inequality of arithmetic-geometric means, we have for any $\gamma_{1}>0$

$$
\begin{array}{r}
2\left(\llbracket \boldsymbol{u}_{h}^{n+1} \rrbracket,\left\{\mu \varepsilon\left(\boldsymbol{u}_{h}^{n+1}\right) \mathbf{n}\right\}\right)_{\Gamma_{\tilde{h}}^{n}} \leq \frac{\gamma_{1} \mu}{h}\left\|\llbracket \boldsymbol{u}_{h}^{n+1} \rrbracket\right\|_{L^{2}\left(\Gamma_{\tilde{h}}^{n}\right)}^{2}+\frac{h \mu}{\gamma_{1}}\left\|\left\{\varepsilon\left(\boldsymbol{u}_{h}^{n+1}\right)\right\}\right\|_{L^{2}\left(K \cap \Gamma_{\tilde{h}}^{n}\right)}^{2} \\
=\frac{\gamma_{1} \mu}{h}\left\|\llbracket \boldsymbol{u}_{h}^{n+1} \rrbracket\right\|_{L^{2}\left(\Gamma_{\tilde{h}}^{n}\right)}^{2}+\sum_{i=1}^{2} \sum_{K \in \mathcal{T}_{i, h}^{n}} \frac{h \mu}{\gamma_{1}}\left\|\varepsilon\left(\boldsymbol{u}_{i, h}^{n+1}\right)\right\|_{L^{2}\left(K \cap \Gamma_{\tilde{h}}^{n}\right)}^{2} .
\end{array}
$$

Now we look to bound the norm of the average of the symmetric gradient over the interface, which has been separated into an interior and exterior component using the triangle inequality. For some function $v \in H^{1}(K)$, with the help of [20, Lemma 1] and noting that the polygonal interface $\Gamma_{\tilde{h}}^{n}$ is Lipschitz,

$$
\begin{aligned}
\|v\|_{L^{2}\left(K \cap \Gamma_{\tilde{h}}^{n}\right)}^{2} & \leq C_{1}\left(h^{-1}\|v\|_{L^{2}(K)}^{2}+h\|\nabla v\|_{L^{2}(K)}^{2}\right) \\
& \leq C_{1} h^{-1}\|v\|_{L^{2}(K)}^{2}+C_{1} \cdot \tilde{C}_{I} h^{-1}\|v\|_{L^{2}(K)}^{2}
\end{aligned}
$$

Here, $C_{1}$ is the constant from [20] and $\tilde{C}_{I}$ is the constant from the well-known finite element inverse inequality

$$
\|\nabla v\|_{L^{2}(K)}^{2} \leq \tilde{C}_{I} h^{-2}\|v\|_{L^{2}(K)}^{2} .
$$

Letting $v$ be each component of the symmetric part of the gradient in (4.9) and adding the resulting inequalities and denote $C_{I}=\tilde{C}_{I}+1$ yields

$$
\left\|\varepsilon\left(\boldsymbol{u}_{i, h}^{n+1}\right)\right\|_{L^{2}\left(K \cap \Gamma_{\tilde{h}}^{n}\right)}^{2} \leq C_{1} C_{I} h^{-1}\left\|\varepsilon\left(\boldsymbol{u}_{i, h}^{n+1}\right)\right\|_{L^{2}(K)}^{2},
$$

and we can control the inner product over $\Gamma_{\tilde{h}}^{n}$ by

$$
\begin{aligned}
\left(\llbracket \boldsymbol{u}_{h}^{n+1} \rrbracket,\left\{\mu \varepsilon\left(\boldsymbol{u}_{h}^{n+1}\right)\right\}\right)_{\Gamma_{\tilde{h}}^{n}} & \leq \frac{\gamma_{1} \mu}{h}\left\|\llbracket \boldsymbol{u}_{h}^{n+1} \rrbracket\right\|_{L^{2}\left(\Gamma_{\tilde{h}}^{n}\right)}^{2}+\frac{C_{1} C_{I}}{\gamma_{1}} \sum_{i=1}^{2} \sum_{K \in \mathcal{T}_{h}} \mu\left\|\varepsilon\left(\boldsymbol{u}_{i, h}^{n+1}\right)\right\|_{L^{2}(K)}^{2} \\
& =\frac{\gamma_{1} \mu}{h}\left\|\llbracket \boldsymbol{u}_{h}^{n+1} \rrbracket\right\|_{L^{2}\left(\Gamma_{\tilde{h}}^{n}\right)}^{2}+\frac{C_{1} C_{I}}{\gamma_{1}} \sum_{i=1}^{2} \mu\left\|\varepsilon\left(\boldsymbol{u}_{i, h}^{n+1}\right)\right\|_{L^{2}\left(\Omega_{i, \tilde{h}}^{n, e}\right)}^{2}
\end{aligned}
$$




$$
\begin{aligned}
\leq \frac{\gamma_{1} \mu}{h}\left\|\llbracket \boldsymbol{u}_{h}^{n+1} \rrbracket\right\|_{L^{2}\left(\Gamma_{\tilde{h}}^{n}\right)}^{2}+\frac{C_{1} C_{I} C_{\boldsymbol{\varepsilon}}}{\gamma_{1}}\left(\mu\left\|\varepsilon\left(\boldsymbol{u}_{h}^{n+1}\right)\right\|_{L^{2}(\Omega)}^{2}\right. \\
\left.+\sum_{i=1}^{2} \mu j_{i, h}\left(\boldsymbol{u}_{i, h}^{n+1}, \boldsymbol{u}_{i, h}^{n+1}\right)\right)
\end{aligned}
$$

where the final inequality is the result of Lemma 4.2. Similarly, we have

$$
2\left(\boldsymbol{u}_{1, h}^{n+1}, \mu \varepsilon\left(\boldsymbol{u}_{1, h}^{n+1}\right)\right)_{\partial \Omega} \leq \frac{\gamma_{1} \mu}{h}\left\|\boldsymbol{u}_{1, h}^{n+1}\right\|_{L^{2}(\partial \Omega)}^{2}+\frac{C_{1} C_{I} C_{\boldsymbol{\varepsilon}}}{\gamma_{1}} \mu\left\|\varepsilon\left(\boldsymbol{u}_{h}^{n+1}\right)\right\|_{L^{2}(\Omega)}^{2} .
$$

Now we combine all of these inequalities and choose $\gamma_{1}$ so that $\frac{2 C_{1} C_{I} C_{\varepsilon}}{\gamma_{1}} \leq 1$ and multiply both sides by $\Delta t$ to get (4.7).

To establish the discrete inf-sup condition for Q2-P1 time-depedent Stokes elements for CutFEM, see [24, 28, 30, 35, 36, 44]. This will be published elsewhere in the context of the CutFEM shown in this work. We note however that to establish energy stability, we only need to control $\left(\llbracket \boldsymbol{u}^{n+1} \rrbracket,\left\{q^{n+1}\right\} \mathbf{n}\right)_{\Gamma_{\tilde{h}}^{n}}=\left(\llbracket \boldsymbol{u}^{n+1} \rrbracket \cdot \mathbf{n},\left\{q^{n+1}\right\}\right)_{\Gamma_{\tilde{h}}^{n}}$. Using similar arguments as in the sources above we have:

1. If $\mu / h$ dominates $h / \Delta t$, then we control via

$$
\left(\llbracket \boldsymbol{u}^{n+1} \rrbracket \cdot \mathbf{n},\left\{q^{n+1}\right\}\right)_{\Gamma_{\tilde{h}}^{n}} \leq \frac{\gamma_{1} \mu}{h}\left\|\llbracket \boldsymbol{u}_{h}^{n+1} \rrbracket\right\|_{L^{2}\left(\Gamma_{\tilde{h}}^{n}\right)}^{2}+\frac{h}{4 \gamma_{1} \mu}\left\|\left\{q^{n+1}\right\}\right\|_{L^{2}\left(\Gamma_{\tilde{h}}^{n}\right)}^{2},
$$

2. If $h / \Delta t$ dominates $\mu / h$, then we control via

$$
\left(\llbracket \boldsymbol{u}^{n+1} \rrbracket \cdot \mathbf{n},\left\{q^{n+1}\right\}\right)_{\Gamma_{\tilde{h}}^{n}} \leq \frac{\gamma_{2} h}{\Delta t}\left\|\llbracket \boldsymbol{u}_{h}^{n+1} \cdot \mathbf{n} \rrbracket\right\|_{L^{2}\left(\Gamma_{\tilde{h}}^{n}\right)}^{2}+\frac{\Delta t}{4 \gamma_{2} h}\left\|\left\{q^{n+1}\right\}\right\|_{L^{2}\left(\Gamma_{\tilde{h}}^{n}\right)}^{2} .
$$

Hence, let us define

$$
\gamma_{p}=\min \left\{\frac{1}{4 \gamma_{1} \mu}, \frac{\Delta t}{4 \gamma_{2} h^{2}}\right\} \quad \text { and } \quad \gamma_{\boldsymbol{u}}=\gamma_{1} \mu+\gamma_{2} \frac{h^{2}}{\Delta t} .
$$

REMARK 4.4. We now highlight the most relevant changes of the discrete formulation in the presence of diffusive coefficient jump across $\Gamma$. We indicate the changes in (4.4a) and (4.4c); see [11, 14, 24]. Let the index "-" be the side where $\mu_{-}=\min \left\{\mu_{1}, \mu_{2}\right\}$. The average $\{\phi\}=\frac{1}{2}\left(\left.\phi\right|_{\Omega_{1}}+\left.\phi\right|_{\Omega_{2}}\right)$ is replaced by $\{\phi\}=\phi_{-}$, and the penalty term $\gamma_{1} \frac{\mu}{h} \int_{\Gamma_{\tilde{h}}^{n}} \llbracket \boldsymbol{u}_{h}^{n+1} \rrbracket^{2} d s$ is replaced by $\gamma_{1} \frac{\mu_{-}}{h} \int_{\Gamma_{\tilde{h}}^{n}} \llbracket \boldsymbol{u}_{h}^{n+1} \rrbracket^{2} d s$. The remaining changes are natural; just take $\mu_{i}$ when the integration is in $\Omega_{i, \tilde{h}}^{n}$.

5. Numerical results. Below we illustrate the theoretical findings and some approximation results with numerical simulations. The numerical test cases confirm the unconditionally energy stability of the semi-implicit method and the conditionally stability of the explicit method.

In each example we choose the computational domain to be the square $\Omega=(0,1)^{2}$ with the fluid initially at rest. Let the reference configuration for $\Gamma$ be the unit interval, i.e., $L=1$. We subdivide the interval $[0,1]$ into $m+1$ equally-spaced points $s_{j}$. Then the step size in $[0,1]$ is $\tilde{h}=1 / m$. Note that the spacing $\tilde{h}$ can be chosen entirely independent of $h$. However, in the numerical experiments that follow, $\tilde{h}$ is selected based on the bulk fluid mesh size $h$ by choosing $m$ such that the initial interface discretization $\left\{\mathbf{X}_{j}^{0}\right\}_{j=0}^{m}:=\left\{\Gamma\left(s_{j}, 0\right)\right\}_{j=0}^{m}$ roughly satisfies

$$
\max _{j}\left|\mathbf{X}_{j+1}^{0}-\mathbf{X}_{j}^{0}\right| \leq h / 2
$$


In other words, the maximum distance between two points in the original discretization is less than or equal to half of the bulk fluid element size. The procedure used to determine $m$ in each example is as follows:

1. fix $h$,

2. discretize $\Gamma(s, 0)$ using a fine spacing $s=0,0.001, \ldots, 1$ to obtain a relatively smooth discretization of the interface,

3. set $m$ by scaling the number of points in the fine discretization by the ratio of the maximum segement length in the fine discretization to the goal maximum segment length $2 h$, i.e.,

$$
m=\left\lceil 1001 \cdot\left(\max _{j=0, \ldots, 1000} \frac{\left|\Gamma\left(s_{j+1}, 0\right)-\Gamma\left(s_{j}, 0\right)\right|}{2 h}\right)\right\rceil .
$$

We choose the penalty parameters from (4.1) and (4.2) to be $\gamma_{1}=\gamma_{2}=10$. With our choice of uniform $\tilde{h}$ we further expect $\Gamma_{\tilde{h}}^{n}$ to approach a regular polygon with the sampled points equally spaced along the interface.

In each example, since the coupled problem can be reduced to a second-order in time partial differential equation, the immersed boundary should oscillat,e and due to the viscosity it should converge to a circular steady state. Due to the incompressibility of the fluid, the interior area enclosed by the membrane should not change in time. Another goal of the numerical tests is to show the good approximation of these properties.

5.1. Example 1: spatial convergence. The results in Table 5.1 illustrate the convergence of our method for the steady-state problem

$$
\begin{aligned}
-\mu \nabla \cdot \boldsymbol{\varepsilon}\left(\boldsymbol{u}_{i}\right)+\nabla p_{i}=\mathbf{f} & \text { in } \Omega_{i}, i=1,2 \\
\nabla \cdot \boldsymbol{u}_{i}=0 & \text { in } \Omega_{i}, i=1,2 .
\end{aligned}
$$

The boundary condition for $\boldsymbol{u}_{1}$ on $\partial \Omega$ and jump conditions $\llbracket(\mu \boldsymbol{\varepsilon}(\boldsymbol{u})-p) \mathbf{n} \rrbracket, \llbracket \boldsymbol{u} \cdot \mathbf{n} \rrbracket$, and $\llbracket \boldsymbol{u} \rrbracket$ on $\Gamma$ are chosen to match the exact test solutions

$$
\begin{aligned}
& \boldsymbol{u}_{1}=\left[\begin{array}{c}
\sin (x) \cos (y) \\
-\cos (x) \sin (y)
\end{array}\right], \quad p_{1}=\sin (2 \pi x) \cos (2 \pi y), \\
& \boldsymbol{u}_{2}=\left[\begin{array}{c}
x e^{-x y} \\
-y e^{-x y}
\end{array}\right], \quad p_{2}=x^{2} y^{2} .
\end{aligned}
$$

The exact solution in (5.2) exhibits nonzero jumps in the velocity and stress across the interface, independent of our choice of $\Gamma$. Table 5.1 was generated using a circular interface of radius $r=0.3$ centered at $(0.5,0.5)$. The discrete interface $\Gamma_{\tilde{h}}$ was constructed by choosing the reference configuration to be the unit interval, i.e., $L=1$, with $\tilde{h}=1 / 400$ (i.e., $m=400$ ). The $H^{1}$ error observed in these tables is optimal since we are using Q2-P1 elements. Note that the rates $k$ seen in Table 5.1 correspond to the convergence rate $O\left(h^{k}\right)$. We also see superconvergence in the $L^{2}$ - and $H^{1}$-norms of the velocity and near-optimal convergence in the other norms.

To compute the $L^{2}$ - and $H^{1}$-error, we extend $\boldsymbol{u}$ to $\Omega_{i, \tilde{h}}$ when necessary using (5.2) and compute the norms of the difference $\boldsymbol{u}_{i}-\boldsymbol{u}_{i, h}$ on each subdomain. The $L^{\infty}$ - and $W^{1, \infty}$-norms are computed using the difference $\boldsymbol{u}_{i}-\boldsymbol{u}_{i, h}$ at all the nodes where the degrees of freedom of $\boldsymbol{u}_{h}$ is imposed, and also include the points on $\Gamma_{\tilde{h}}$, including each $s_{j}$ and all points where $\Gamma_{\tilde{h}}$ intersects edges of elements in $\mathcal{T}_{i, h}$ by interpolating $\boldsymbol{u}_{h}$. 


\section{ETNA}

Kent State University and Johann Radon Institute (RICAM)

TABLE 5.1

Error tables for (a) the velocity and (b) the pressure solving (5.1) in Example 1. The numbers shown are the values of the difference between the approximate solution and the exact solution given by (5.2) in the specified norm with spatial grid size $h$. Each rate $k$ corresponds to the convergence rate $O\left(h^{k}\right)$.

(a) Error of the velocity.

\begin{tabular}{l|cc|cc|cc|cc}
$1 / h$ & $L^{2}$ & $k$ & $H^{1}$ & $k$ & $L^{\infty}$ & $k$ & $W^{1, \infty}$ & $k$ \\
\hline 8 & $6.0751 \mathrm{e}-4$ & & $1.2079 \mathrm{e}-2$ & & $2.9443 \mathrm{e}-3$ & & $1.5822 \mathrm{e}-1$ & \\
16 & $5.7992 \mathrm{e}-5$ & 3.4 & $2.8194 \mathrm{e}-3$ & 2.1 & $4.0194 \mathrm{e}-4$ & 2.9 & $5.0981 \mathrm{e}-2$ & 1.6 \\
32 & $4.0155 \mathrm{e}-6$ & 3.9 & $4.7479 \mathrm{e}-4$ & 2.6 & $4.6180 \mathrm{e}-5$ & 3.1 & $1.3094 \mathrm{e}-2$ & 2.0 \\
64 & $3.8898 \mathrm{e}-7$ & 3.4 & $8.4839 \mathrm{e}-5$ & 2.5 & $7.5565 \mathrm{e}-6$ & 2.6 & $3.7782 \mathrm{e}-3$ & 1.8 \\
128 & $3.0663 \mathrm{e}-8$ & 3.7 & $1.5375 \mathrm{e}-5$ & 2.5 & $9.9287 \mathrm{e}-7$ & 3.0 & $1.0938 \mathrm{e}-3$ & 1.8
\end{tabular}

(b) Error of the pressure.

\begin{tabular}{r|cc|lc|cc|cc}
$1 / h$ & $L^{2}$ & $k$ & $H^{1}$ & $k$ & $L^{\infty}$ & $k$ & $W^{1, \infty}$ & $k$ \\
\hline 8 & $3.7455 \mathrm{e}-2$ & & 1.3695 & & $2.3560 \mathrm{e}-1$ & & 6.5007 & \\
16 & $7.1874 \mathrm{e}-3$ & 2.4 & $6.1616 \mathrm{e}-1$ & 1.2 & $5.2798 \mathrm{e}-2$ & 2.2 & 2.9639 & 1.1 \\
32 & $1.7328 \mathrm{e}-3$ & 2.1 & $3.0661 \mathrm{e}-1$ & 1.0 & $1.6484 \mathrm{e}-2$ & 1.7 & 2.0968 & 0.5 \\
64 & $4.1940 \mathrm{e}-4$ & 2.0 & $1.5074 \mathrm{e}-1$ & 1.0 & $5.4817 \mathrm{e}-3$ & 1.6 & 1.3588 & 0.6 \\
128 & $1.0151 \mathrm{e}-4$ & 2.0 & $7.4491 \mathrm{e}-2$ & 1.0 & $1.4711 \mathrm{e}-3$ & 1.9 & $7.4067 \mathrm{e}-1$ & 0.9
\end{tabular}

TABLE 5.2

Normalized deviation of the interior area at $t=0.5$ from the initial interior area in Example 2. Results obtained using $\mu=1$ and $\kappa=10$ with $\Delta t$ and $h$ as shown.

\begin{tabular}{cc|cccc} 
& & \multicolumn{4}{c}{$\Delta t$} \\
$1 / h$ & 16 & $-1.6336 \mathrm{e}-04$ & $-8.0403 \mathrm{e}-05$ & $-3.8474 \mathrm{e}-05$ & $-1.7869 \mathrm{e}-05$ \\
\cline { 2 - 6 } & 32 & $-1.9443 \mathrm{e}-04$ & $-1.0152 \mathrm{e}-04$ & $-5.4441 \mathrm{e}-05$ & $-2.9699 \mathrm{e}-05$ \\
& 64 & $-1.8896 \mathrm{e}-04$ & $-9.6995 \mathrm{e}-05$ & $-5.1840 \mathrm{e}-05$ & $-2.8784 \mathrm{e}-05$
\end{tabular}

5.2. Example 2: ellipse. The second example is a common scenario found in the related literature $[7,32]$. The interface $\Gamma$ will begin as an ellipse where the initial points chosen are sampled from

$$
\mathbf{X}^{0}(s)=\left[\begin{array}{l}
0.3 \cos (2 \pi s)+0.5 \\
0.4 \sin (2 \pi s)+0.5
\end{array}\right], \quad s \in[0,1]
$$

The discrete interface $\Gamma_{\tilde{h}}^{0}$ is approximated by mapping $m+1$ equally-spaced points from $[0,1]$, where $m=162$ for $N \stackrel{h}{=} 32$ in this example. It is worth noting that the lengths of two adjacent segments on $\Gamma_{\tilde{h}}^{0}$ may be different. Since $\tilde{h}$ is chosen to be constant across each reference segment throughout all simulations, in addition to bending, the result is also a "tension" force, or a stretching in the direction tangent to $\Gamma_{\tilde{h}}^{0}$. The effects of such a force will be emphasized in Example 4; see Figure 5.9.

As seen in Figure 5.1, the solution computed using the explicit method, with parameters chosen such that an instability occurs, blows up very quickly as the energy fails to dissipate. With a smaller time step, we see a more gradual increase in energy as the method does not fail so quickly. The semi-implicit method exhibits the theoretical energy stability over the explicit method and remains stable with each set of parameters tested. Figure 5.2 shows the position of the interface at three time steps capturing one intermediate step before steady state is achieved prior to $t=1$. 


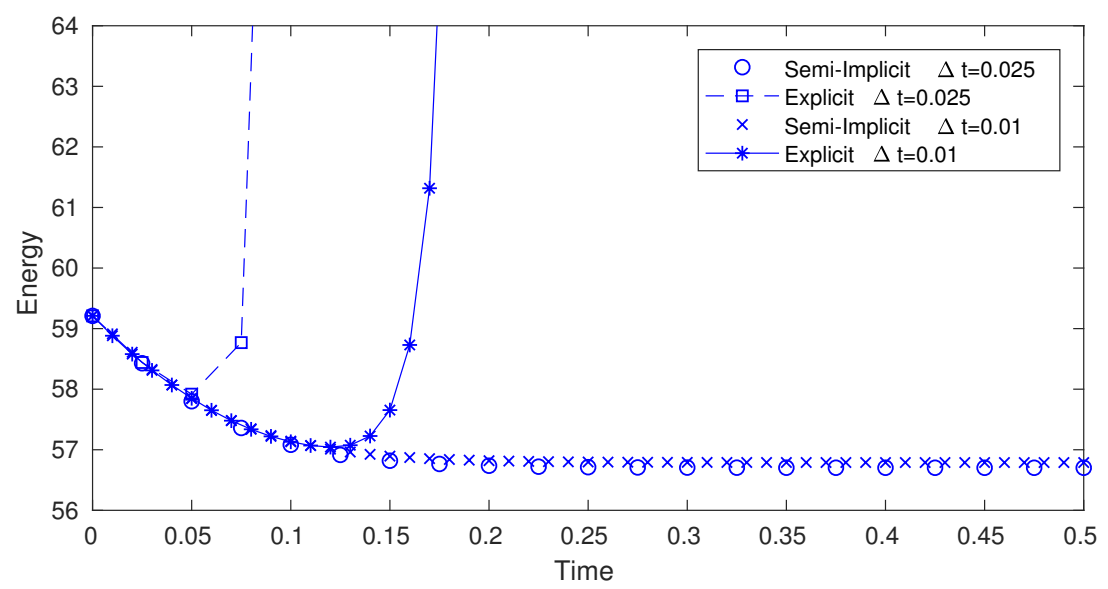

FIG. 5.1. Plot of the energy of the system in Example 2 comparing the explicit and semi-implicit methods with $\mu=1, \kappa=12, h=1 / 32$, and $\Delta t=0.05$ ( $\square$ for explicit, $\circ$ for semi-implicit) or $\Delta t=0.01$ ( $*$ for explicit, $\times$ for semi-implicit). The lines connecting data points are included when plotting the results of the explicit method to highlight the steep increase in energy.

Table 5.2 provides the normalized deviation at time $t=0.5$ from the original interior area. Due to the incompressibility of the fluid, the optimal result is a constant interior area as the interface moves. Recall that $m$ is the number of points sampled from $\Gamma^{0}$ to form the polygon $\Gamma_{\tilde{h}}^{0}$. As the mesh size $h$ decreases, we increase $m$. In addition to improving the initial approximation of each subdomain, the conservation of the interior becomes more accurate as the mesh is refined. We also see significant improvement in the conservation of interior area as $\Delta t$ is refined. In Table 5.2 we see that the deviation from the interior area is no larger than $0.05 \%$ with the chosen parameters, and can be reduced to less than $0.008 \%$ by refining $h$ and $\Delta t$. It is worth noting that in this example a greater improvement is seen by reducing $\Delta t$ compared to reducing $h$.

We now turn to some observations of the temporal convergence of the semi-implicit method. In Table 5.3 the convergence of fluid velocity in the $L^{2}$-norm over the domain $\Omega$ is estimated using Richardson extrapolation. The value of the ratio shown in the table corresponds to

$$
r_{\Delta t}=\log _{2}\left(\frac{\left\|\boldsymbol{u}_{\Delta t}-\boldsymbol{u}_{\Delta t / 2}\right\|_{L^{2}(\Omega)}}{\left\|\boldsymbol{u}_{\Delta t / 2}-\boldsymbol{u}_{\Delta t / 4}\right\|_{L^{2}(\Omega)}}\right),
$$

where $\boldsymbol{u}_{\Delta t}$ is the approximation of $\boldsymbol{u}$ at $t=0.1$ computed using time step $\Delta t$. The convergence of the method can be seen to be of order $O\left(\Delta t^{r}\right)$. Since the method is high-order in $h$, the error is dominated by time discretization errors, and Table 5.3 shows that the error of the fluid velocity in the $L^{2}$-norm is asymptotically linear in $\Delta t$.

Figure 5.3 displays the point-wise linear convergence of the interior and exterior traction to $\Gamma_{\tilde{h}}$. The traction is computed at the midpoint of each segment of $\Gamma_{\tilde{h}}$, corresponding to $s_{j+\frac{1}{2}}$ in the reference configuration because the normal vector is not well-defined at each vertex of the polygon $\Gamma_{\tilde{h}}$. In each plot the traction at $t=0.1$ computed using $\Delta t=0.1 / 2^{6}$ is compared to the traction computed using $\Delta t=0.1 / 2^{3}, \Delta t=0.1 / 2^{4}$, and $\Delta t=0.1 / 2^{5}$. Each line shows the sum of the absolute value of the difference in each component of the traction vector at each point $s_{j+\frac{1}{2}}$ in the reference configuration. Similarly, Figure 5.4 shows the 


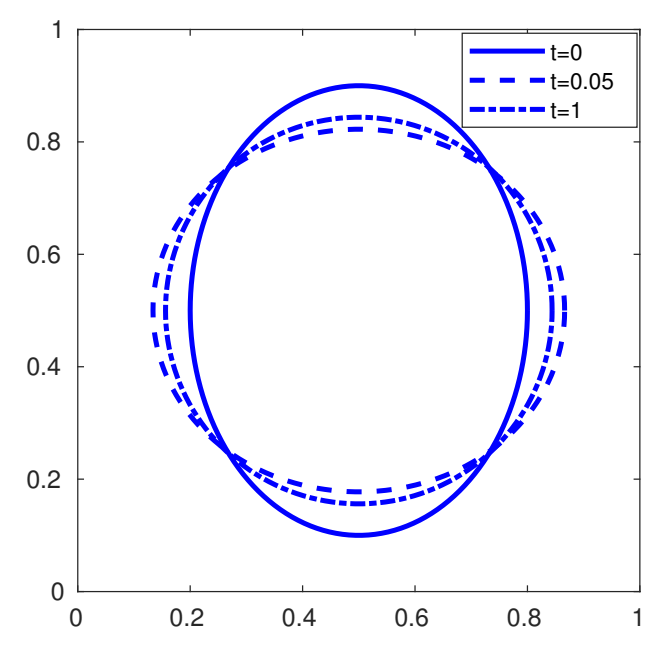

FIG. 5.2. Plot of the position of the interface shown at $t=0, t=0.05$, and $t=1$. Simulation run with $\mu=0.01, \kappa=50, N=32, \Delta t=0.01$, and the initial configuration given in Example 2.

TABLE 5.3

Temporal convergence rates $r_{\Delta t}$ using Richardson extrapolation for Example 2 (ellipse) and Example 3 (heart) with $\mu=10$ and $\kappa=1$. The results from this test indicate linear convergence of velocity $\boldsymbol{u}$ in the $L^{2}$-norm when using the semi-implicit method.

\begin{tabular}{c|ccccc|c}
\multicolumn{7}{c}{$\Delta t$} \\
& 0.1 & $0.1 / 2$ & $0.1 / 2^{2}$ & $0.1 / 2^{3}$ & $0.1 / 2^{4}$ & Avg. \\
\hline Ellipse & 1.0165 & 0.9905 & 1.3825 & 1.9083 & 0.5673 & 1.1730 \\
Heart & 3.7075 & 2.3262 & -0.9455 & 4.7735 & 1.2742 & 2.2272
\end{tabular}

linear convergence in the interface location. The interface location at $t=0.1$ computed using $\Delta t=0.1 / 2^{6}$ is compared to the traction computed using $\Delta t=0.1 / 2^{3}, \Delta t=0.1 / 2^{4}$, and $\Delta t=0.1 / 2^{5}$. The sum of the absolute value of the difference in each coordinate $\mathbf{X}\left(s_{j}, 0.1\right)$ is plotted.

5.3. Example 3: heart. The third example is used to ensure that energy stability still holds regardless of the convexity of the interface and displacement of the centroid of the interior subdomain. The original curve is constructed as the sum of two translated cardioids and is parameterized by $s \in[0,1]$ as follows:

$$
\mathbf{X}^{0}(s)=\frac{1}{20}\left[\begin{array}{l}
\cos (2 \pi s)(7(1-\sin (2 \pi s))+3(1-\cos (2 \pi s)))+24 \\
\sin (2 \pi s)(3(1-\sin (2 \pi s))+7(1-\cos (2 \pi s)))+24
\end{array}\right] .
$$

Again, the discrete initial interface $\Gamma_{\tilde{h}}^{0}$ is approximated using $m+1$ equally-spaced points from $[0,1]$, however, now we let $m=186$ for $N=32$.

The energy plots in Figure 5.5 show that the energy in the explicit method becomes unstable slower than in the previous example. However, the semi-implicit method remains stable. Figure 5.6 displays the position of the interface as it deforms and moves toward the top-right corner of $\Omega$, approaching a circular steady state. In this figure we observe a quick deformation to a convex interior at $t=0.05$ and a translation of this region in the subsequent time steps. Table 5.4 gives the normalized deviation from the original interior area at time 


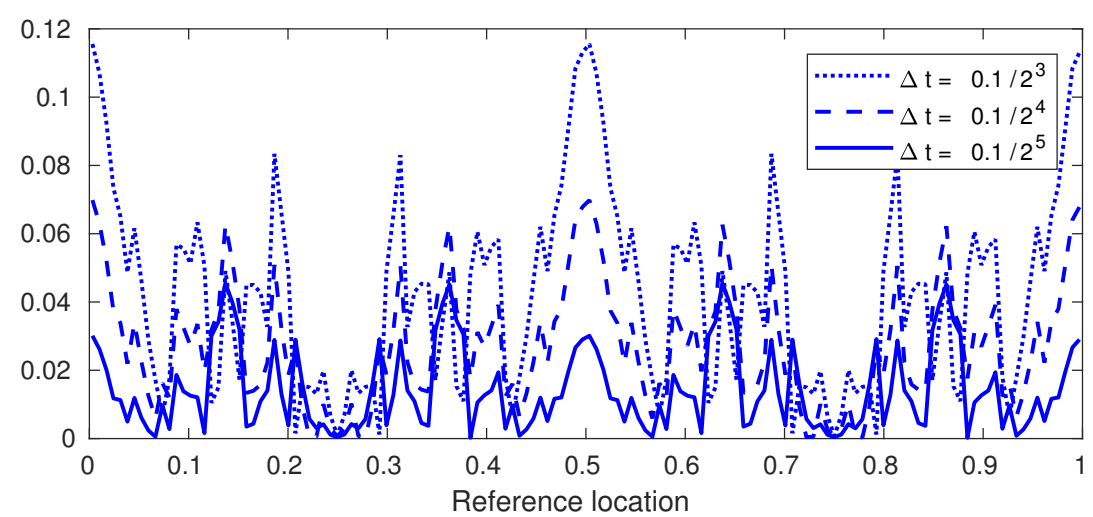

(a) Interior traction.

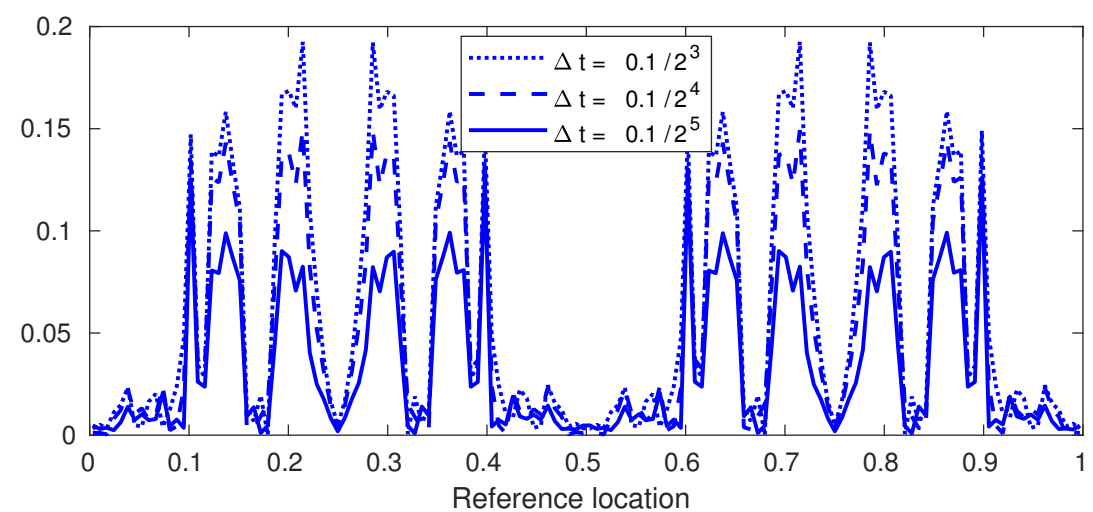

(b) Exterior traction.

FIG. 5.3. The above figure illustrates the convergence of the traction at $t=0.1$ to the "true" solutions $\boldsymbol{u}_{i}^{*}$ and $p_{i}^{*}$ computed using $\Delta t=0.1 / 2^{6}$. Parameters used are $\mu=0.1$ and $\kappa=1$. The initial interface configuration is that described in Example 2. The quantity plotted is $\sum_{i=1}^{2}\left|\left(\boldsymbol{\varepsilon}\left(\boldsymbol{u}_{i}\right)-p_{i}\right)-\left(\boldsymbol{\varepsilon}\left(\boldsymbol{u}_{i}^{*}\right)-p_{i}^{*}\right)\right|$ evaluated at each midpoint on $\Gamma_{\tilde{h}}$, where $\boldsymbol{u}_{i}$ and $p_{i}$ are solved using the $\Delta t$ specified in the legend. Point-wise convergence of the interior and exterior traction is observed.

$t=0.5$. Similar to the previous example, we see more improvement from a reduction of $\Delta t$ than from a refinement of $h$. Here, the area loss is reduced almost linearly with the reduction in $\Delta t$ and very little corresponding to a smaller mesh size $h$.

In Table 5.3 the temporal convergence of the velocity in the $L^{2}$-norm is estimated using Richardson extrapolation alongside the rate of convergence estimates for the previous example. One can see that the rate of convergence is super-linear in both examples.

Figure 5.7 illustrates the point-wise convergence of the interior and exterior traction. The traction is evaluated at the midpoint of each segment of $\Gamma_{\tilde{h}}$, and the quantity plotted is analogous to that in the previous example. Figure 5.8 displays the convergence of the interface at $t=0.1$ using refined values of $\Delta t$ compared to the interface location at $t=0.1$ using $\Delta t=0.1 / 2^{6}$.

5.4. Example 4: stretched circle. The fourth example is chosen to emphasize the effects of a nonuniform tension around the perimeter of $\Gamma_{\tilde{h}}^{0}$. The initial configuration is a circle of 


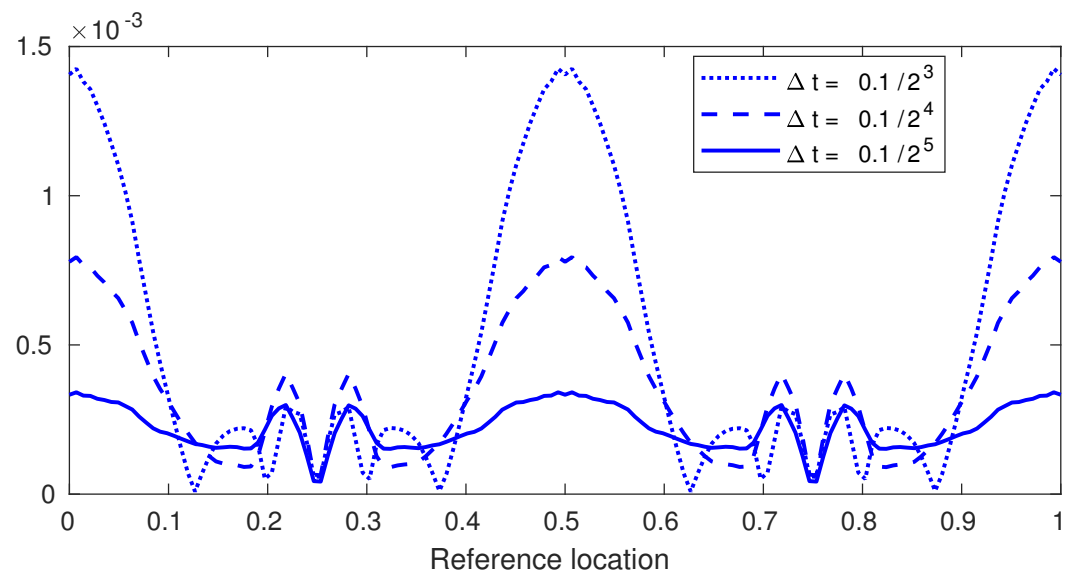

FIG. 5.4. Plot of the difference between the interface location at $t=0.1$ computed using the time step indicated in the plot and the "true" solution computed using $\Delta t=0.1 / 2^{6}$. Parameters used are $\mu=0.1$ and $\kappa=1$, and the initial interface configuration is that described in Example 2. The quantity plotted is the sum of the absolute value of the difference at each midpoint on $\Gamma_{\tilde{h}}$.

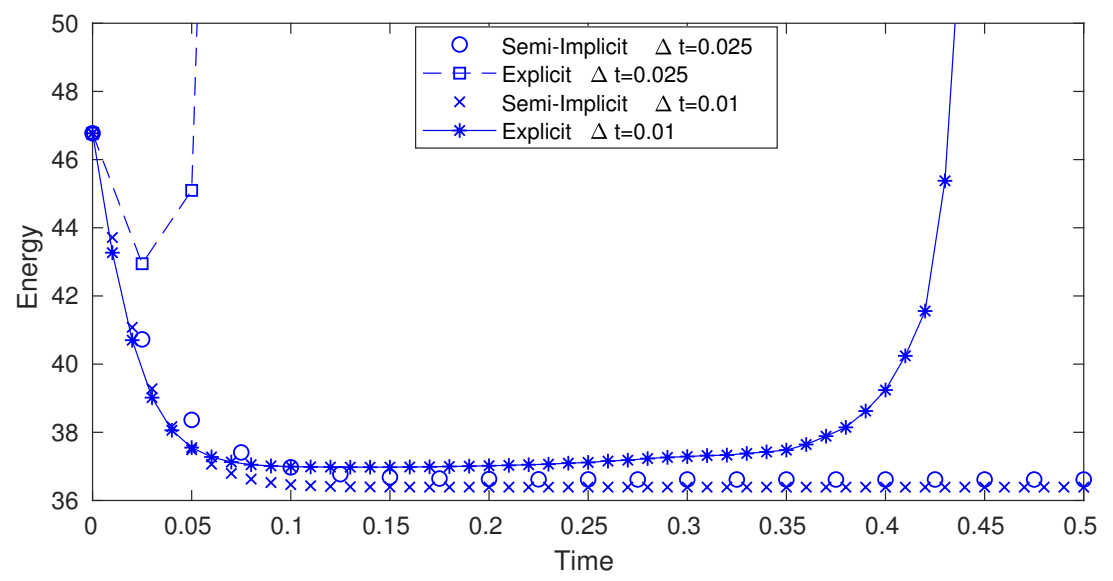

FIG. 5.5. Plot of the energy of the system in Example 3, comparing the explicit and semi-implicit methods with $\mu=1, \kappa=12, N=32$, and $\Delta t=0.025$ ( $\square$ for explicit, $\circ$ for semi-implicit) or $\Delta t=0.01$ ( $*$ for explicit, $\times$ for semi-implicit). The lines connecting data points are included when plotting the results of the explicit method to highlight the steep increase in energy.

TABLE 5.4

Normalized deviation of the interior area at $t=0.5$ from the initial interior area in Example 3. Results obtained using $\mu=1$ and $\kappa=10$ with $\Delta t$ and $h$ as shown.

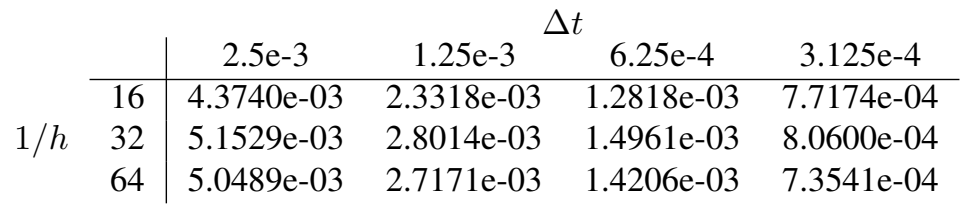




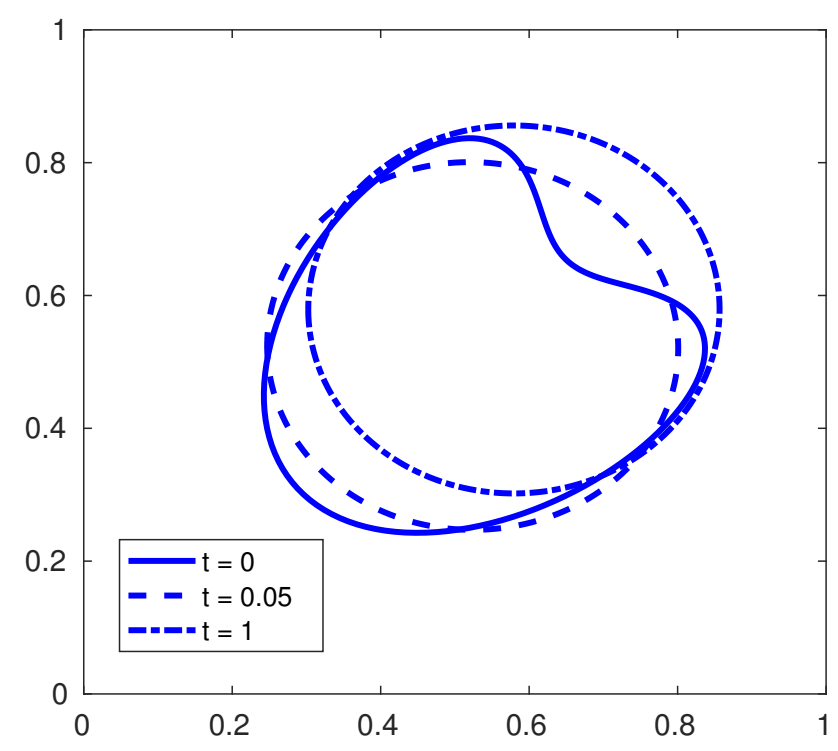

FIG. 5.6. Plot of the position of the interface shown at $t=0, t=0.05$, and $t=1$. Simulation run with $\mu=0.01, \kappa=50, N=32, \Delta t=0.01$, and the initial configuration from Example 3 .

radius $\frac{1}{4}$ centered at $\left(\frac{1}{2}, \frac{1}{2}\right)$ with its parameterization given by

$$
\mathbf{X}^{0}(s)=\frac{1}{4}\left[\begin{array}{l}
\cos (2 \pi s)+2 \\
\sin (2 \pi s)+2
\end{array}\right], \quad s \in[0,1] .
$$

Previously we chose equally-spaced points from the interval $[0, L]$ when the tension of each segment was arbitrary. Since $\Gamma_{\tilde{h}}^{0}$ is a circle, we must sample at nonuniform $s_{j}$ to prescribe a nonuniform tension on the edges of the polygon $\Gamma_{\tilde{h}}^{0}$. The parameter values $\tilde{s}_{j}$ used to compute $\mathbf{X}_{j}^{0}=\mathbf{X}^{0}\left(\tilde{s}_{j}\right)$ are the $m+1$ evenly spaced points $s_{j} \in[0,1]$ mapped by the cubic function

$$
\tilde{s}_{j}=\frac{1}{5}\left(16 s_{j}^{3}-24 s_{j}^{2}+13 s_{j}\right) .
$$

The result of this simulation, computed using the semi-implicit method, will be a leftward moving circle as a force tangent to the interface is applied to the fluid. Equilibrium is obtained when the points on the circle are equally spaced and the total force applied to the fluid is zero. Figure 5.9 displays the position of the points on the interface at three time steps. These plots highlight the leftward motion and the even distribution of the points on $\Gamma_{\tilde{h}}^{n}$ near the steady state at $t=1$. In this example, the semi-implicit method is unconditionally stable.

6. Conclusions. In this work, we presented a new finite element method for solving the unsteady Stokes equations with an immersed membrane that moves with the velocity of the fluid, not known a priori. We successfully combined the classical immersed boundary method with Nitsche's formulation and CutFEM to solve this problem in two dimensions. The proposed method maintains the use of the Dirac delta function to pass the force applied by the immersed structure in the Lagrangian frame to the fluid in the Eulerian frame. We developed a semi-implicit discretization and added the necessary consistent penalty terms to 


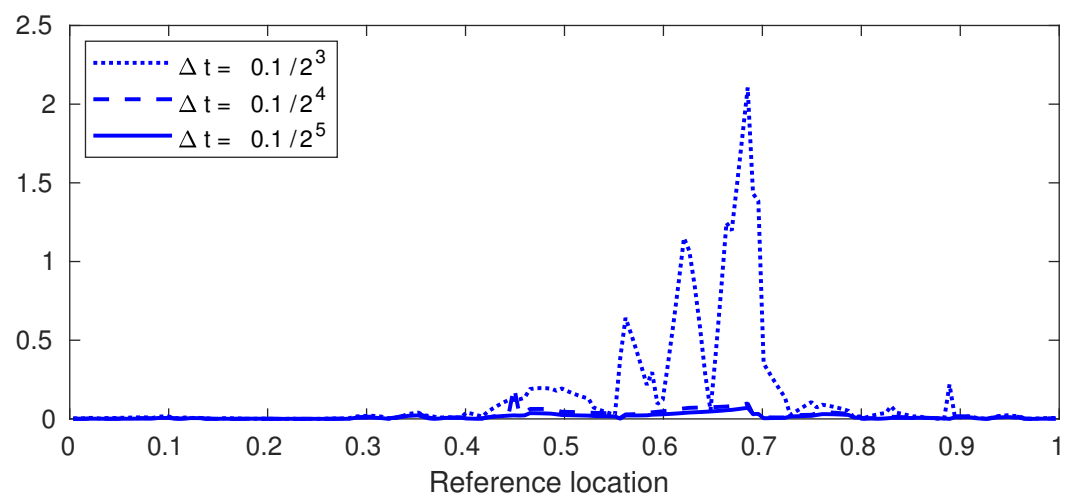

(a) Interior traction.

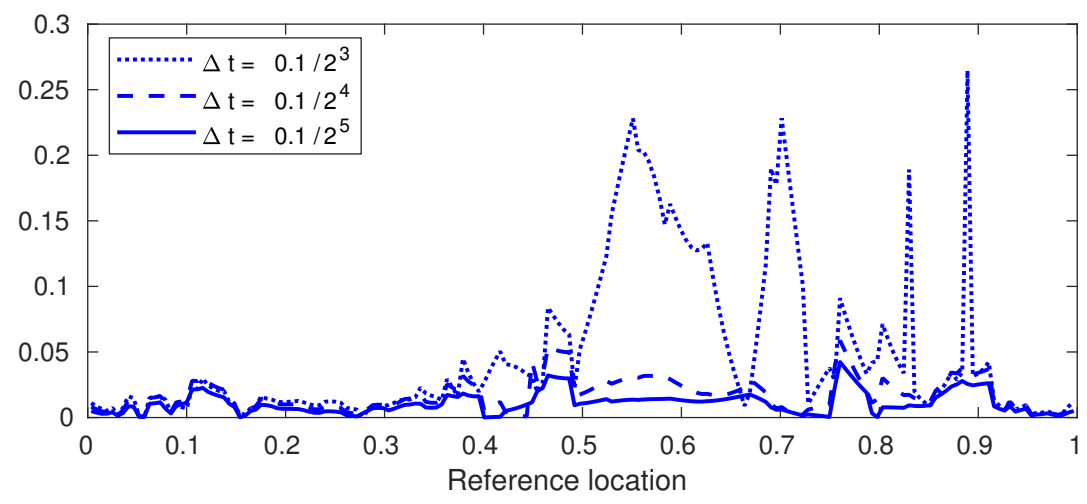

(b) Exterior traction.

FIG. 5.7. The above figure illustrates the convergence of the traction at $t=0.1$ to the "true" solutions $\boldsymbol{u}_{i}^{*}$ and $p_{i}^{*}$ computed using $\Delta t=0.1 / 2^{6}$. Parameters are set to $\mu=10$ and $\kappa=1$. The initial interface configuration is that described in Example 3. The quantity plotted is $\sum_{i=1}^{2}\left|\left(\varepsilon\left(\boldsymbol{u}_{i}\right)-p_{i}\right)-\left(\varepsilon\left(\boldsymbol{u}_{i}^{*}\right)-p_{i}^{*}\right)\right|$ evaluated at each midpoint on $\Gamma_{\tilde{h}}$, where $\boldsymbol{u}_{i}$ and $p_{i}$ are solved using the $\Delta$ t specified in the legend. Point-wise convergence of the interior and exterior traction is observed.

maintain energy stability. The stability of our method is proved and verified in each example of our numerical results. This semi-implicit method was tested alongside the explicit CutFEM method, which is the algorithm directly analogous to the original finite element immersed boundary method [5]. Using CutFEM we improved the spatial convergence of the velocity to second-order, compared to existing first-order methods, of the fluid near the interface compared to existing methods $[5,6]$; however, we continued the use of a polygonal approximation to the interface for its simplicity in computing the location of $\Gamma_{\tilde{h}}^{n+1}$.

The numerical results demonstrate that if the polygonal approximation to the interface is refined as the mesh is refined, we obtain optimal spatial convergence in Example 1, as shown in Table 5.1. We also observe in Examples 2- 4 the theoretical unconditional energy stability proved in this work. The conservation of area in each subdomain is desired and obtained for sufficiently small values of $h$ and $\Delta t$. The trends observed in Table 5.2 and Table 5.4 are seen for sufficiently small values of $\Delta t$. As we refine $\Delta t$, we see an improvement in area conservation as $\Delta t$ is refined; however, we may not observe such trends as $h$ is reduced.

The backward Euler scheme was shown to be first-order in time. Higher-order in time 


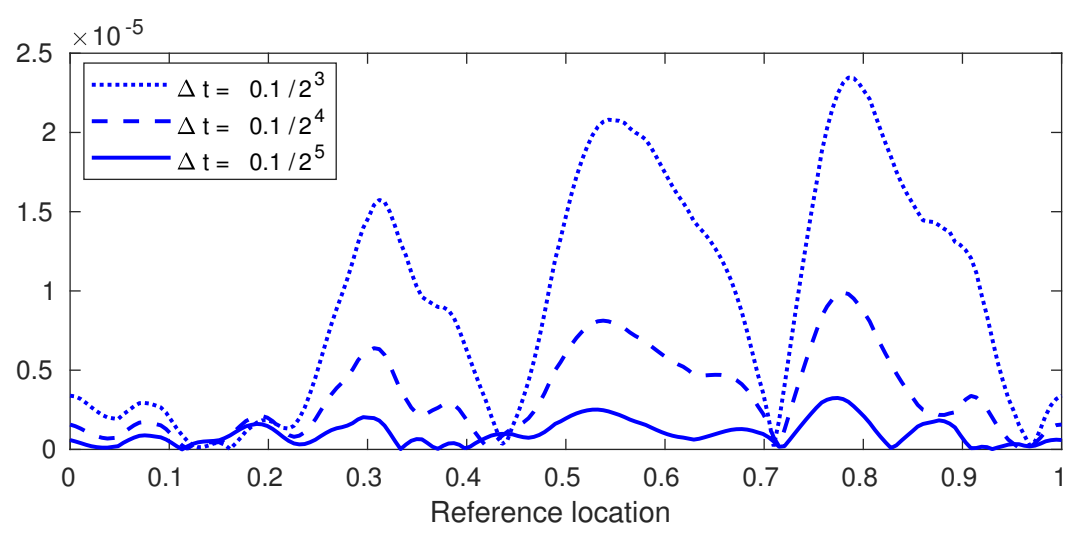

FIG. 5.8. Plot of the difference between the interface location at $t=0.1$ computed the time steps indicated in the plot and the "true" solution computed using $\Delta t=0.1 / 2^{6}$. Parameters used are $\mu=10$ and $\kappa=1$, and the initial interface configuration is that described in Example 3. The quantity plotted is the sum of the absolute value of the difference at each point midpoint on $\Gamma_{\tilde{h}}$.
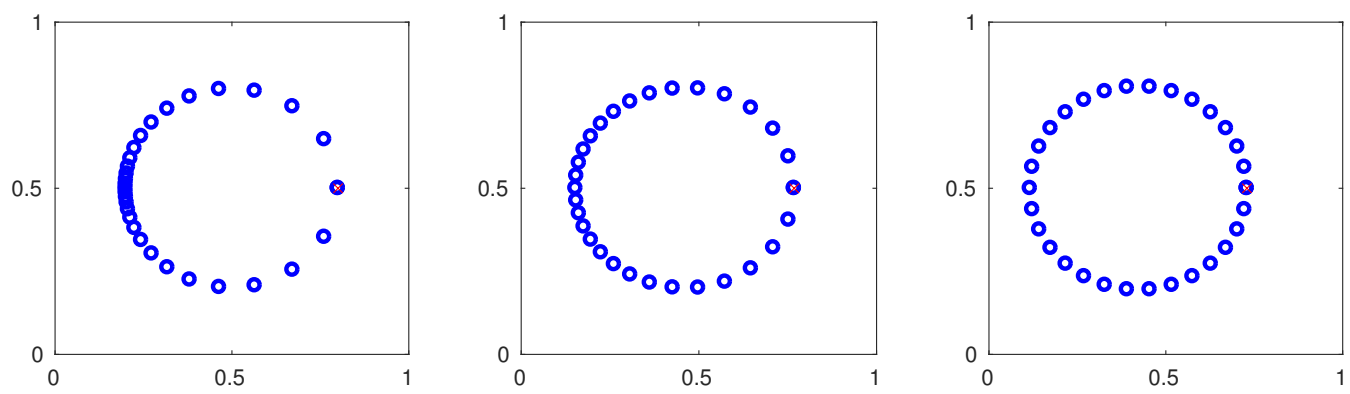

FIG. 5.9. Position of the interface at $t=0, t=0.05$, and $t=1$. We let $\mu=1, \kappa=10, \Delta t=0.01$, and $N=32$ and use the initial configuration from Example 4. An encircled red $\times$ is used to denote $\mathbf{X}\left(s_{0}, t\right)$ for reference. We display every 6 th point on the interface to reduce clutter.

methods are an interesting topic for future work. The extension to higher-order finite differences and Crank-Nicolson schemes are nontrivial and require further investigation. In particular, $\tilde{\boldsymbol{u}}$ defined in the beginning of Section 3 should be defined in a different way to preserve incompressibility.

\section{REFERENCES}

[1] S. AdJerid, M. BEN-Romdhane, AND T. Lin, Higher degree immersed finite element methods for secondorder elliptic interface problems, Int. J. Numer. Anal. Model., 11 (2014), pp. 541-566.

[2] G. Agresar, J. J. Linderman, G. Tryggvason, And K. G. Powell, An adaptive, Cartesian, fronttracking method for the motion, deformation and adhesion of circulating cells, J. Comput. Phys., 143 (1998), pp. 346-380.

[3] E. BÄNSCH, Finite element discretization of the Navier-Stokes equations with a free capillary surface, Numer. Math., 88 (2001), pp. 203-235.

[4] R. BECKER, E. BURMAN, AND P. HANSBO, A Nitsche extended finite element method for incompressible elasticity with discontinuous modulus of elasticity, Comput. Methods Appl. Mech. Engrg., 198 (2009), pp. 3352-3360.

[5] D. BOFFI AND L. GASTALDI, A finite element approach to the immersed boundary method, Comput. \& Structures, 81 (2003), pp. 491-501. 
[6] D. Boffi, L. Gastaldi, And L. Heltai, Numerical stability of the finite element immersed boundary method, Math. Models Methods Appl. Sci., 17 (2007), pp. 1479-1505.

[7] D. Boffi, L. Gastaldi, L. Heltai, And C. S. Peskin, On the hyper-elastic formulation of the immersed boundary method, Comput. Methods Appl. Mech. Engrg., 197 (2008), pp. 2210-2231.

[8] E. Burman, Ghost penalty, C. R. Math. Acad. Sci. Paris, 348 (2010), pp. 1217-1220.

[9] E. Burman, S. Claus, P. Hansbo, M. G. Larson, And A. Massing, CutFEM: discretizing geometry and partial differential equations, Internat. J. Numer. Methods Engrg., 104 (2015), pp. 472-501.

[10] E. BURMAN AND M. A. FernÁNDEZ, Stabilization of explicit coupling in fluid-structure interaction involving fluid incompressibility, Comput. Methods Appl. Mech. Engrg., 198 (2009), pp. 766-784.

[11] E. BuRman, J. GUZMÁn, M. A. SÁNCHEZ, AND M. SARKIS, Robust flux error estimation of Nitsche's method for high contrast interface problems, IMA J. Numer. Anal., 38 (2018), pp. 646-668.

[12] E. BURMAN AND P. HANSBO, Fictitious domain finite element methods using cut elements: I. A stabilized Lagrange multiplier method, Comput. Methods Appl. Mech. Engrg., 199 (2010), pp. 2680-2686.

[13] _ Fictitious domain finite element methods using cut elements: II. A stabilized Nitsche method, Appl. Numer. Math., 62 (2012), pp. 328-341.

[14] E. CÁCERES, J. GUZMÁN, AND M. OLSHANSKII, New stability estimates for an unfitted finite element method for two-phase Stokes problem, SIAM J. Numer. Anal., 58 (2020), pp. 2165-2192.

[15] J. CheSSA AND T. BELYTSCHKo, An extended finite element method for two-phase fluids, Trans. ASME J. Appl. Mech., 70 (2003), pp. 10-17.

[16] T. FRACHON AND S. ZAHEDI, A cut finite element method for incompressible two-phase Navier-Stokes flows, J. Comput. Phys., 384 (2019), pp. 77-98.

[17] Y. GONG, B. LI, AND Z. LI, Immersed-interface finite-element methods for elliptic interface problems with nonhomogeneous jump conditions, SIAM J. Numer. Anal., 46 (2007/08), pp. 472-495.

[18] Y. GONG AND Z. LI, Immersed interface finite element methods for elasticity interface problems with nonhomogeneous jump conditions, Numer. Math. Theory Methods Appl., 3 (2010), pp. 23-39.

[19] S. GRoss AND A. ReUSKen, Numerical Methods for Two-Phase Incompressible Flows, Springer, Berlin, 2011.

[20] J. GuZmÁn And M. Olshanskit, Inf-sup stability of geometrically unfitted Stokes finite elements, Math. Comp., 87 (2018), pp. 2091-2112.

[21] J. GUZMÁn, M. A. SÁNCHEZ, AND M. SARKIS, On the accuracy of finite element approximations to a class of interface problems, Math. Comp., 85 (2016), pp. 2071-2098.

[22] _ - A finite element method for high-contrast interface problems with error estimates independent of contrast, J. Sci. Comput., 73 (2017), pp. 330-365.

[23] P. HANSBO, Nitsche's method for interface problems in computational mechanics, GAMM-Mitt., 28 (2005), pp. 183-206.

[24] P. HANSBo, M. G. LARSON, AND S. ZAHEDI, A cut finite element method for a Stokes interface problem, Appl. Numer. Math., 85 (2014), pp. 90-114.

[25] — A cut finite element method for coupled bulk-surface problems on time-dependent domains, Comput. Methods Appl. Mech. Engrg., 307 (2016), pp. 96-116.

[26] X. HE, T. LIN, AND Y. LIN, Immersed finite element methods for elliptic interface problems with nonhomogeneous jump conditions, Int. J. Numer. Anal. Model., 8 (2011), pp. 284-301.

[27] _ The convergence of the bilinear and linear immersed finite element solutions to interface problems, Numer. Methods Partial Differential Equations, 28 (2012), pp. 312-330.

[28] Q. HONG AND J. KRAUS, Uniformly stable discontinuous Galerkin discretization and robust iterative solution methods for the Brinkman problem, SIAM J. Numer. Anal., 54 (2016), pp. 2750-2774.

[29] M.-C. LAI AND Z. LI, A remark on jump conditions for the three-dimensional Navier-Stokes equations involving an immersed moving membrane, Appl. Math. Lett., 14 (2001), pp. 149-154.

[30] R. LAZAROV AND A. TAKHIROV, A note on the uniform inf-sup condition for the Brinkman problem in highly heterogeneous media, J. Comput. Appl. Math., 340 (2018), pp. 537-545.

[31] R. J. LEVEQUE AND Z. LI, The immersed interface method for elliptic equations with discontinuous coeffcients and singular sources, SIAM J. Numer. Anal., 31 (1994), pp. 1019-1044.

[32] _ _ Immersed interface methods for Stokes flow with elastic boundaries or surface tension, SIAM J. Sci. Comput., 18 (1997), pp. 709-735.

[33] Z. LI, T. LIN, AND X. WU, New Cartesian grid methods for interface problems using the finite element formulation, Numer. Math., 96 (2003), pp. 61-98.

[34] T. LIN, Y. LIN, AND X. ZHANG, Partially penalized immersed finite element methods for elliptic interface problems, SIAM J. Numer. Anal., 53 (2015), pp. 1121-1144.

[35] K. A. MARDAL, X.-C. TAI, AND R. WINTHER, A robust finite element method for Darcy-Stokes flow, SIAM J. Numer. Anal., 40 (2002), pp. 1605-1631.

[36] K.-A. MARDAL AND R. WINTHER, Uniform preconditioners for the time dependent Stokes problem, Numer. Math., 98 (2004), pp. 305-327. 


\section{ETNA}

Kent State University and

Johann Radon Institute (RICAM)

[37] A. Massing, M. G. Larson, A. Logg, and M. E. Rognes, A stabilized Nitsche fictitious domain method for the Stokes problem, J. Sci. Comput., 61 (2014), pp. 604-628.

[38] E. P. Newren, A. L. Fogelson, R. D. Guy, And R. M. Kirby, Unconditionally stable discretizations of the immersed boundary equations, J. Comput. Phys., 222 (2007), pp. 702-719.

[39] J. NITSCHE, Über ein Variationsprinzip zur Lösung von Dirichlet-Problemen bei Verwendung von Teilräumen, die keinen Randbedingungen unterworfen sind, Abh. Math. Sem. Univ. Hamburg, 36 (1971), pp. 9-15.

[40] C. S. PESKIn, Numerical analysis of blood flow in the heart, J. Comput. Phys., 25 (1977), pp. 220-252.

[41] - The immersed boundary method, Acta Numer., 11 (2002), pp. 479-517.

[42] C. S. PESKIN AND D. M. MCQUEEN, A three-dimensional computational method for blood flow in the heart. I. Immersed elastic fibers in a viscous incompressible fluid, J. Comput. Phys., 81 (1989), pp. 372-405.

[43] C. TU AND C. S. PESkin, Stability and instability in the computation of flows with moving immersed boundaries: a comparison of three methods, SIAM J. Sci. Statist. Comput., 13 (1992), pp. 1361-1376.

[44] X. XIE, J. XU, AND G. XUE, Uniformly-stable finite element methods for Darcy-Stokes-Brinkman models, J. Comput. Math., 26 (2008), pp. 437-455. 